\title{
Averaging principle for a class of stochastic reaction-diffusion equations *
}

\author{
Sandra Cerrai \\ Dip. di Matematica per le Decisioni \\ Università di Firenze \\ Via C. Lombroso 6/17 \\ I-50134 Firenze, Italy
}

\author{
Mark Freidlin \\ Department of Mathematics \\ University of Maryland \\ College Park \\ Maryland, USA
}

\begin{abstract}
We consider the averaging principle for stochastic reaction-diffusion equations. Under some assumptions providing existence of a unique invariant measure of the fast motion with the frozen slow component, we calculate limiting slow motion. The study of solvability of Kolmogorov equations in Hilbert spaces and the analysis of regularity properties of solutions, allow to generalize the classical approach to finite-dimensional problems of this type in the case of SPDE's.
\end{abstract}

\section{Introduction}

Consider a Hamiltonian system with one degree of freedom. In the area where the Hamiltonian has no critical points, one can introduce action-angle coordinates $(I, \varphi)$, with $I \in \mathbb{R}^{1}$ and $0 \leq \varphi \leq 2 \pi$, so that the system has the form

$$
\dot{I}_{t}=0, \quad \dot{\varphi}_{t}=\omega\left(I_{t}\right) .
$$

Now, consider small perturbations of this system such that, after an appropriate time rescaling, the perturbed system can be written as follows

$$
\dot{I}_{t}^{\epsilon}=\beta_{1}\left(I_{t}^{\epsilon}, \varphi_{t}^{\epsilon}\right), \quad \dot{\varphi}_{t}^{\epsilon}=\frac{1}{\epsilon} \omega\left(I_{t}^{\epsilon}\right)+\beta_{2}\left(I_{t}^{\epsilon}, \varphi_{t}^{\epsilon}\right) .
$$

Here the perturbations $\beta_{1}, \beta_{2}: \mathbb{R}^{1} \times[0,2 \pi] \rightarrow \mathbb{R}$ are assumed to be regular enough functions, as well as $\omega: \mathbb{R} \rightarrow \mathbb{R}$, and $0<\epsilon<<1$.

System (1.2) has a fast component, which is, roughly speaking, the motion along the non-perturbed trajectories (1.1), after the time change $t \rightarrow t / \epsilon$, and the slow component which can be described by the evolution of $I_{t}^{\epsilon}$. When $\epsilon$ goes to 0 , the slow component approaches the averaged motion $\bar{I}_{t}$, defined by

$$
\dot{\bar{I}}_{t}=\bar{\beta}_{1}\left(\bar{I}_{t}\right), \quad \bar{I}_{0}=I_{0},
$$

${ }^{*}$ Key words and phrases: Stochastic reaction diffusion equations, invariant measures and ergodicity, averaging principle, Kolmogorov equations in Hilbert spaces. 
where

$$
\bar{\beta}_{1}(y)=\frac{1}{2 \pi} \int_{0}^{2 \pi} \beta_{1}(y, \varphi) d \varphi .
$$

This is a classical manifestation of the averaging principle for equation (1.2).

To prove the convergence of $I_{t}^{\epsilon}$ to $\bar{I}_{t}$, one can consider a $2 \pi$-periodic in $\varphi$ solution $u(I, \varphi)$ of an auxiliary equation

$$
\mathcal{L}^{I} u(I, \varphi):=\omega(I) \frac{\partial u}{\partial \varphi}=\beta_{1}(I, \varphi)-\bar{\beta}(I)
$$

It is easy to see that such a solution exists and is unique up to an additive function, depending just on $I$. Moreover, it can be chosen in such a way that $u(I, \varphi)$ has continuous derivatives in $I$ and $\varphi$. Actually, $u(I, \varphi)$ can be written explicitly. It follows from (1.2) and (1.4) that

$$
\begin{aligned}
& u\left(I_{t}^{\epsilon}, \varphi_{t}^{\epsilon}\right)-u\left(I_{0}^{\epsilon}, \varphi_{0}^{\epsilon}\right)=\frac{1}{\epsilon} \int_{0}^{t} \frac{\partial u}{\partial \varphi}\left(I_{s}^{\epsilon}, \varphi_{s}^{\epsilon}\right) \omega\left(I_{s}^{\epsilon}\right) d s+\int_{0}^{t} \frac{\partial u}{\partial \varphi}\left(I_{s}^{\epsilon}, \varphi_{s}^{\epsilon}\right) \beta_{2}\left(I_{s}^{\epsilon}, \varphi_{s}^{\epsilon}\right) d s \\
& +\int_{0}^{t} \frac{\partial u}{\partial I}\left(I_{s}^{\epsilon}, \varphi_{s}^{\epsilon}\right) \beta_{1}\left(I_{s}^{\epsilon}, \varphi_{s}^{\epsilon}\right) d s=\frac{1}{\epsilon} \int_{0}^{t}\left[\beta_{1}\left(I_{s}^{\epsilon}, \varphi_{s}^{\epsilon}\right)-\bar{\beta}\left(I_{s}^{\epsilon}\right)\right] d s \\
& +\int_{0}^{t} \frac{\partial u}{\partial \varphi}\left(I_{s}^{\epsilon}, \varphi_{s}^{\epsilon}\right) \beta_{2}\left(I_{s}^{\epsilon}, \varphi_{s}^{\epsilon}\right) d s+\int_{0}^{t} \frac{\partial u}{\partial I}\left(I_{s}^{\epsilon}, \varphi_{s}^{\epsilon}\right) \beta_{1}\left(I_{s}^{\epsilon}, \varphi_{s}^{\epsilon}\right) d s .
\end{aligned}
$$

Hence, by taking into account the boundedness of coefficients $\beta_{1}$ and $\beta_{2}$ and of function $u(I, \varphi)$ together with its first derivatives, one can conclude from the last equality that for any $T>0$

$$
\sup _{0 \leq t \leq T}\left|\int_{0}^{t}\left[\beta_{1}\left(I_{s}^{\epsilon}, \varphi_{s}^{\epsilon}\right)-\bar{\beta}\left(I_{s}^{\epsilon}\right)\right] d s\right| \leq c \epsilon,
$$

for some constant $c>0$. Now, from (1.2) and (1.3) it follows

$$
I_{t}^{\epsilon}-\bar{I}_{t}=\int_{0}^{t}\left[\beta_{1}\left(I_{s}^{\epsilon}, \varphi_{s}^{\epsilon}\right)-\bar{\beta}\left(I_{s}^{\epsilon}\right)\right] d s+\int_{0}^{t}\left[\bar{\beta}\left(I_{s}^{\epsilon}\right)-\bar{\beta}\left(\bar{I}_{s}\right)\right] d s,
$$

so that, assuming that $\beta(I, \varphi)$ (and thus $\bar{\beta}(I))$ is Lipschitz-continuous, thanks to (1.5) and to Gronwall's lemma we get

$$
\sup _{0 \leq t \leq T}\left|I_{t}^{\epsilon}-\bar{I}_{t}\right| \leq c \epsilon
$$

On a first glance, one can think that consideration of the auxiliary equation (1.4) for proving averaging principle is an artificial trick. But, actually, this is not the case; the use of equation (1.4) and its natural generalizations helps to prove averaging principle in many cases. For example, when deterministic perturbations of a completely integrable system with many degrees of freedom (in a domain where one can introduce action-angle coordinates) are considered, the operator $\mathcal{L}$ is the generator of the corresponding flow on a torus. Because of the existence of resonance tori, where invariant measure of the flow is not unique, one has to consider approximate solutions of the corresponding equation (1.4). The price for this is that the convergence of $\sup _{0 \leq t \leq T}\left|I_{t}^{\epsilon}-\bar{I}_{t}\right|$ to zero does not hold for any 
fixed initial condition, but just in Lebesgue measure in the phase space, given that the set of resonance tori is small enough (see [18]). An approximate solution of the corresponding analogue of equation (1.4) is used in [9] for averaging of stochastic perturbations. In this case it is possible to prove weak convergence to the averaged system in the space of continuous functions on the phase space. Moreover, concerning the use of the auxiliary equation (1.4), it is worthwhile mentioning that in [19] suitable correction functions arising as solutions of problems analogous to (1.4) are introduced in order to prove some limit theorems for more general multi-scaling systems.

An analogue of equation (1.4) appears also in the case when the fast motion is a stochastic process

$$
\dot{I}_{t}^{\epsilon}=\beta_{1}\left(I_{t}^{\epsilon}, \varphi_{t}^{\epsilon}\right), \quad \dot{\varphi}_{t}^{\epsilon}=\frac{1}{\epsilon} \omega\left(I_{t}^{\epsilon}, \varphi_{t}^{\epsilon}\right)+\frac{1}{\sqrt{\epsilon}} \sigma\left(I_{t}^{\epsilon}, \varphi_{t}^{\epsilon}\right) \dot{w}_{t}+\beta_{2}\left(I_{t}^{\epsilon}, \varphi_{t}^{\epsilon}\right) .
$$

Here $I, \varphi:[0,+\infty) \rightarrow \mathbb{R}^{n}, \omega: \mathbb{R}^{n} \times \mathbb{R}^{n} \rightarrow \mathbb{R}^{n}, \sigma(I, \varphi) \sigma^{*}(I, \varphi)=\alpha(I, \varphi)$ is a positive definite $n \times n$-matrix and $w_{t}$ is the standard $n$-dimensional Wiener process. All functions are assumed to be $2 \pi$-periodic in the variables $\varphi_{i}$ and smooth enough. Under these conditions, for each $I \in \mathbb{R}^{n}$ the diffusion process $\varphi_{t}^{I}$ on the $n$-torus $T^{n}$ defined by the equation

$$
\dot{\varphi}_{t}^{I}=\omega\left(I, \varphi_{t}^{I}\right)+\sigma\left(I, \varphi_{t}^{I}\right) \dot{w}_{t},
$$

has a unique invariant measure with density $m_{I}(\varphi)$. Then equation (1.4) should be replaced by

$$
\mathcal{L}^{I} u(I, \varphi)=\beta_{1}(I, \varphi)-\bar{\beta}_{1}(I)
$$

where $\mathcal{L}^{I}$ is the generator of the process $\varphi_{t}^{I}$ and for any $I \in \mathbb{R}^{n}$

$$
\bar{\beta}_{1}(I):=\int_{T^{n}} \beta_{1}(I, \varphi) m_{I}(\varphi) d \varphi .
$$

Taking into account the uniqueness of the invariant measure, one can check that there exists a solution to problem (1.6) which is smooth in $I$ and $\varphi$. Applying Itô's formula to $u\left(I_{t}^{\epsilon}, \varphi_{t}^{\epsilon}\right)$, one can prove not just weak convergence of $I_{t}^{\epsilon}$ to $\bar{I}_{t}$ on any finite time interval, but also convergence of $\left(I_{t}^{\epsilon}-\bar{I}_{t}\right) / \sqrt{\epsilon}$ to a diffusion process.

Besides the situations described above, averaging principle both for deterministically and for randomly perturbed systems, having a finite number of degrees of freedom, has been studied by many authors, under different assumptions and with different methods. The first rigorous results are due to Bogoliubov (see [2]). Further developments were obtained by Volosov, Anosov and Neishtadt (see [18] and [22]) and by Arnold et al. (see [1]). All these references are for the deterministic case. Concerning the stochastic case, it is worth quoting the paper by Khasminskii [11], the works of Brin, Freidlin and Wentcell (see [3, [7], [8], [9]), Veretennikov (see [21]) and Kifer (see for example [12, [13], [14], 15]).

To the best of our knowledge, very few has been done as far as averaging for infinite dimensional systems is concerned. To this purpose we recall the papers [17] and [20], where the case of stochastic evolution equations in abstract Hilbert spaces is considered, and the paper [16], where randomly perturbed KdV equation is studied. 
In this paper we are dealing with a system of reaction-diffusion equations with a stochastic fast component. Namely, for each $0<\epsilon<<1$, we consider the system of partial differential equations

$$
\left\{\begin{array}{l}
\frac{\partial u^{\epsilon}}{\partial t}(t, \xi)=\mathcal{A} u^{\epsilon}(t, \xi)+f\left(\xi, u^{\epsilon}(t, \xi), v^{\epsilon}(t, \xi)\right), \quad t \geq 0, \quad \xi \in[0, L], \\
\frac{\partial v^{\epsilon}}{\partial t}(t, \xi)=\frac{1}{\epsilon}\left[\mathcal{B} v^{\epsilon}(t, \xi)+g\left(\xi, u^{\epsilon}(t, \xi), v^{\epsilon}(t, \xi)\right)\right]+\frac{1}{\sqrt{\epsilon}} \frac{\partial w}{\partial t}(t, \xi), \quad t \geq 0, \quad \xi \in[0, L], \\
u^{\epsilon}(0, \xi)=x(\xi), \quad v^{\epsilon}(0, \xi)=y(\xi), \quad \xi \in[0, L], \\
\mathcal{N}_{1} u^{\epsilon}(t, \xi)=\mathcal{N}_{2} v^{\epsilon}(t, \xi)=0, \quad t \geq 0, \quad \xi \in\{0, L\} .
\end{array}\right.
$$

The present model describes a typical and relevant situation for reaction-diffusion systems in which the diffusion coefficients and the rates of reactions have different order. In the case we are considering here, the noise is included only in the fast motion and it is of additive type. However, we would like to stress that the introduction of a noisy term of additive type in the slow equation would not lead to any new effects, as it should be included in the limiting slow motion without any substantial changes.

The linear operators $\mathcal{A}$ and $\mathcal{B}$, appearing respectively in the slow and in the fast equation, are second order uniformly elliptic operators and $\mathcal{N}_{1}$ and $\mathcal{N}_{2}$ are some operators acting on the boundary. The operator $\mathcal{B}$, endowed with the boundary conditions $\mathcal{N}_{2}$, is self-adjoint and strictly dissipative (see Hypothesis 1 ).

The reaction coefficients $f$ and $g$ are measurable mappings from $[0, L] \times \mathbb{R}^{2}$ into $\mathbb{R}$ which satisfy suitable regularity assumptions and for the reaction coefficient $g$ in the fast motion equation some dissipativity assumption is assumed (see Hypotheses 2 and 3).

The noisy perturbation of the fast motion equation is given by a space-time white noise $\partial w / \partial t(t, \xi)$, defined on a complete stochastic basis $\left(\Omega, \mathcal{F}, \mathcal{F}_{t}, \mathbb{P}\right)$.

The corresponding fast motion $v^{x, y}(t)$, with frozen slow component $x \in H:=L^{2}(0, L)$, (the counterpart of the process $\varphi_{t}^{I}$ above in the case of a system with a finite number of degrees of freedom) is a Markov process in a functional space. Notice that the phase space of $v^{x, y}(t)$ is not just infinitely dimensional but also not compact. Nevertheless, by assuming that the system has certain dissipativity properties, for any fixed $x \in H$ the process $v^{x, y}(t)$ has a unique invariant measure $\mu^{x}$. If $\mathcal{L}^{x}$ is the generator of this process, then the counterpart of equation (1.4) has the form

$$
c(\epsilon) \Phi_{h}^{\epsilon}(x, y)-\mathcal{L}^{x} \Phi_{h}^{\epsilon}(x, y)=\langle F(x, y)-\bar{F}(x), h\rangle_{H}, \quad x, y, h \in H,
$$

where $c(\epsilon)$ is a constant depending on $\epsilon$ and vanishing at $\epsilon=0$,

$$
F(x, y)(\xi)=f(\xi, x(\xi), y(\xi)), \quad \xi \in[0, L]
$$

and

$$
\bar{F}(x):=\int_{H} F(x, y) \mu^{x}(d y), \quad x \in H
$$


Notice that in (1.8) we cannot consider the Poisson equation $(c(\epsilon)=0)$, but we have to add a zero order term $c(\epsilon) \Phi_{h}^{\epsilon}(x, y)$, in order to get bounds for $\Phi_{h}^{\epsilon}(x, y)$ and its derivatives which are uniform with respect to $\epsilon \in(0,1]$.

Due to the ergodicity of $\mu^{x}$, we prove that there exists some $\delta>0$ such that for any $\varphi: H \rightarrow \mathbb{R}$ and $x, y \in H$

$$
\left|P_{t}^{x} \varphi(y)-\int_{H} \varphi(z) \mu^{x}(d z)\right| \leq c\left(1+|x|_{H}+|y|_{H}\right) e^{-\delta t}[\varphi]_{\text {Lip }},
$$

where $P_{t}^{x}$ is the transition semigroup associated with the fast motion $v^{x, y}(t)$, with frozen slow component $x$. This implies that the solution $\Phi_{h}^{\epsilon}(x, y)$ of equation (1.8) can be written explicitly as

$$
\Phi_{h}^{\epsilon}(x, y)=\int_{0}^{t} e^{-c(\epsilon) t} P_{t}^{x}\langle F(x, \cdot)-\bar{F}(x), h\rangle_{H}(y) d t .
$$

By using some techniques developed in [4], we obtain bounds for $\Phi_{h}^{\epsilon}(x, y)$ and its derivatives, which in general are not uniform in $x, y \in H$, as the reaction coefficients $f$ and $g$ are not assumed to be bounded. Moreover, we are able to apply an infinite dimensional Itô's formula to $\Phi_{h}^{\epsilon}\left(u^{\epsilon}, P_{n} v^{\epsilon}\right)$, where $P_{n}$ is the projection of $H$ onto the $n$-dimensional space generated by the first $n$ modes of the operator $\mathcal{B}$, and $u^{\epsilon}$ and $v^{\epsilon}$ are the solutions of system (1.7). In this way, as in the case of a system with a finite number of degrees of freedom, we are able to prove that

$$
\mathbb{E}\left|\int_{0}^{t}\left\langle F\left(u^{\epsilon}(s), v^{\epsilon}(s)\right)-\bar{F}\left(u^{\epsilon}(s)\right), h\right\rangle_{H} d s\right| \leq K_{t}(\epsilon), \quad t \geq 0, \quad \epsilon>0,
$$

for some $K_{t}(\epsilon) \downarrow 0$, as $\epsilon$ goes to zero. The proof of (1.9) is one of the major task of the paper, as it requires several estimates for $\Phi_{h}^{\epsilon}(x, y)$ and its derivatives and uniform bounds with respect to $\epsilon>0$, both for $u^{\epsilon}$ and for $v^{\epsilon}$.

Once we have estimate (1.9), we show that for any $T>0$ the family $\left\{\mathcal{L}\left(u^{\epsilon}\right)\right\}_{\epsilon \in(0,1)}$ is tight in $\mathcal{P}\left(C((0, T] ; H) \cap L^{\infty}(0, T ; H)\right)$ and then we identify the weak limit of any subsequence of $\left\{u^{\epsilon}\right\}$ with the solution $\bar{u}$ of the averaged equation

$$
d \bar{u}(t)=A \bar{u}(t)+\bar{F}(\bar{u}(t)), \quad \bar{u}(0)=x \in H .
$$

Now, as a consequence of the dependence of $\mu^{x}$ on $x \in H$, the nonlinear term $\bar{F}$ in (1.10) is a functional of $\bar{u}$. Nevertheless, one can prove that problem (1.10), under certain small assumptions, has a unique solution (see section 5. Proposition 5.1). Hence, by a uniqueness argument, this allows us to conclude that the whole sequence $\left\{u^{\epsilon}\right\}_{\epsilon>0}$ converges to $\bar{u}$ in probability, uniformly on any finite time interval $[0, T]$. That is

Theorem 1.1. Under Hypotheses 1, 2 and 3 (see Section 2), for any $T>0, x, y \in$ $W^{\alpha, 2}(0, L)$, with $\alpha>0$, and $\eta>0$ it holds

$$
\lim _{\epsilon \rightarrow 0} \mathbb{P}\left(\sup _{t \in[0, T]}\left|u^{\epsilon}(t)-\bar{u}(t)\right|_{H}>\eta\right)=0 .
$$


Notice that in the case of space dimension $d=1$ the fast equation with frozen slow component is a gradient system and then we have an explicit expression for the invariant measure $\mu^{x}$. This allows to prove that the mapping $\bar{F}$ is differentiable and to give an expression for its derivative. In such a way we can study dependence with respect to $x$ for the correction function $\Phi_{h}^{\epsilon}$ and we can proceed with the use of Itô's formula. In the case of space dimension $d>1$ the fast equation is no more of gradient type. Nevertheless, in Section 6 we show how it is possible to overcome this difficulty and how, by a suitable approximation procedure, it is still possible to prove averaging.

Finally, we would like to recall that in a number of models, one can assume that the noise in the fast motion is small. This results in replacement of $\epsilon^{-1 / 2}$ by $\delta \epsilon^{-1 / 2}$, with $0<\delta<<1$ (to this purpose we refer to [7]). Then, in generic situation, the invariant measure of the fast motion with frozen slow component $x$ is concentrated, as $\delta$ goes to zero, near one point $y^{\star}(x) \in H$. This is a result of large deviations bounds and $y^{\star}(x)$ can be found as an extremal of a certain functional. In particular, if the operator $\mathcal{B}$ in the fast equation is self-adjoint and $g(\xi, \sigma, \rho)=h(\sigma) N(\rho)$, with, for brevity, the antiderivative $H(\sigma)$ of $h(\sigma)$ having a unique maximum point, then $y^{\star}(x)$ is a constant providing the maximum of $H(\sigma)$. In this case we have that $\bar{F}(x)(\xi)=f\left(\xi, x(\xi), y^{\star}(x)(\xi)\right), \xi \in[0, \pi]$, and (1.10) is a classical reaction-diffusion equation. We will address this problem somewhere else.

\section{Assumptions and notations}

Let $H$ be the Hilbert $L^{2}(0, L)$, endowed with the usual scalar product $\langle\cdot, \cdot\rangle_{H}$ and the corresponding norm $|\cdot|_{H}$. In what follows, we shall denote by $\mathcal{L}(H)$ the Banach space of bounded linear operators on $H$, endowed with the usual sup-norm. $\mathcal{L}_{1}(H)$ denotes the Banach space of trace-class operators, endowed with the norm

$$
\|A\|_{1}:=\operatorname{Tr}\left[\sqrt{A A^{\star}}\right],
$$

and $\mathcal{L}_{2}(H)$ denotes the Hilbert space of Hilbert-Schmidt operators on $H$, endowed with the scalar product

$$
\langle A, B\rangle_{2}=\operatorname{Tr}\left[A B^{\star}\right]
$$

and the corresponding norm $\|A\|_{2}=\sqrt{\operatorname{Tr}\left[A A^{\star}\right]}$.

The Banach space of bounded Borel functions $\varphi: H \rightarrow \mathbb{R}$, endowed with the sup-norm

$$
\|\varphi\|_{0}:=\sup _{x \in H}|\varphi(x)|,
$$

will be denoted by $B_{b}(H) . C_{b}(H)$ is the subspace of all uniformly continuous mappings and $C_{b}^{k}(H)$ is the subspace of all $k$-times differentiable mappings, having bounded and uniformly continuous derivatives, up to the $k$-th order, for $k \in \mathbb{N}$. $C_{b}^{k}(H)$ is a Banach space endowed with the norm

$$
\|\varphi\|_{k}:=\|\varphi\|_{0}+\sum_{i=1}^{k} \sup _{x \in H}\left|D^{i} \varphi(x)\right|_{\mathcal{L}^{i}(H)}=:\|\varphi\|_{0}+\sum_{i=1}^{k}[\varphi]_{i},
$$

where $\mathcal{L}^{1}(H):=H$ and, by recurrence, $\mathcal{L}^{i}(H):=\mathcal{L}\left(H, \mathcal{L}^{i-1}(H)\right)$, for any $i>1$. 
In what follows we shall denote by $\operatorname{Lip}(H)$ the set of all Lipschitz-continuous mappings $\varphi: H \rightarrow \mathbb{R}$ and we shall set

$$
[\varphi]_{\text {Lip }}:=\sup _{x \neq y} \frac{|\varphi(x)-\varphi(y)|}{|x-y|}
$$

Moreover, for any $k \geq 1$ we shall denote by $\operatorname{Lip}^{k}(H)$ the subset of all $k$-times differentiable mappings having bounded and uniformly continuous derivatives, up to the $k$-th order. Notice that for any $\varphi \in \operatorname{Lip}(H)$

$$
|\varphi(y)| \leq[\varphi]_{\text {Lip }}|y|_{H}+|\varphi(0)|, \quad y \in H .
$$

The stochastic perturbation in the fast motion equation is given by a space-time white noise $\partial w / \partial t(t, \xi)$, for $t \geq 0$ and $\xi \in[0, L]$. Formally the cylindrical Wiener process $w(t, \xi)$ is defined as the infinite sum

$$
w(t, \xi)=\sum_{k=1}^{\infty} e_{k}(\xi) \beta_{k}(t), \quad t \geq 0, \quad \xi \in[0, L],
$$

where $\left\{e_{k}\right\}_{k \in \mathbb{N}}$ is a complete orthonormal basis in $H$ and $\left\{\beta_{k}(t)\right\}_{k \in \mathbb{N}}$ is a sequence of mutually independent standard Brownian motions defined on the same complete stochastic basis $\left(\Omega, \mathcal{F}, \mathcal{F}_{t}, \mathbb{P}\right)$.

Now, for any $T>0$ and $p \geq 1$ we shall denote by $\mathcal{H}_{T, p}$ the space of adapted processes in $C\left((0, T] ; L^{p}(\Omega ; H)\right) \cap L^{\infty}\left(0, T ; L^{p}(\Omega ; H)\right) . \mathcal{H}_{T, p}$ is a Banach space, endowed with the norm

$$
\|u\|_{\mathcal{H}_{T, p}}=\left(\sup _{t \in[0, T]} \mathbb{E}|u(t)|_{H}^{p}\right)^{\frac{1}{p}} .
$$

Moreover, we shall denote by $\mathcal{C}_{T, p}$ the subspace of processes $u \in L^{p}(\Omega ; C((0, T] ; H) \cap$ $\left.L^{\infty}(0, T ; H)\right)$, endowed with the norm

$$
\|u\|_{\mathcal{C}_{T, p}}=\left(\mathbb{E} \sup _{t \in[0, T]}|u(t)|_{H}^{p}\right)^{\frac{1}{p}} .
$$

The linear operators $\mathcal{A}$ and $\mathcal{B}$, appearing respectively in the slow and in the fast motion equation, are second order uniformly elliptic operators, having continuous coefficients on $[0, L]$, and $\mathcal{N}_{1}$ and $\mathcal{N}_{2}$ can be either the identity operator (Dirichlet boundary conditions) or first order operators of the following type

$$
\beta(\xi) \frac{\partial}{\partial \xi}+\gamma(\xi), \quad \xi \in\{0, L\}
$$

for some $\beta, \gamma \in C^{1}[0, L]$ such that $\beta(\xi) \neq 0$, for $\xi=0, L$.

As known, the realizations $A$ and $B$ in $H$ of the second order operators $\mathcal{A}$ and $\mathcal{B}$, endowed respectively with the boundary condition $\mathcal{N}_{1}$ and $\mathcal{N}_{2}$, generate two analytic semigroups, which will be denoted by $e^{t A}$ and $e^{t B}, t \geq 0$. Their domains $D(A)$ and $D(B)$ are given by

$$
W_{\mathcal{N}_{i}}^{2,2}(0, L):=\left\{x \in W^{2,2}(0, L): \mathcal{N}_{i} x(0)=\mathcal{N}_{i} x(L)=0\right\}, \quad i=1,2 .
$$


By interpolation we have that for any $0 \leq r \leq s \leq 1 / 2$ and $t>0$ the semigroups $e^{t A}$ and $e^{t B} \operatorname{map} W^{r, 2}(0, L)$ into $W^{s, 2}(0, L) \sqrt{1}$ and

$$
\left|e^{t A} x\right|_{s, 2}+\left|e^{t B} x\right|_{s, 2} \leq c_{r, s}(t \wedge 1)^{-\frac{s-r}{2}} e^{\gamma_{r, s} t}|x|_{r, 2}
$$

for some constants $c_{r, s} \geq 1$ and $\gamma_{r, s} \in \mathbb{R}$.

In what follows, we shall assume that the operator $B$ arising in the fast motion equation fulfills the following condition.

Hypothesis 1. There exists a complete orthonormal basis $\left\{e_{k}\right\}_{k \in \mathbb{N}}$ in $H$ and a sequence $\left\{\alpha_{k}\right\}_{k \in \mathbb{N}}$ such that $B e_{k}=-\alpha_{k} e_{k}$ and

$$
\lambda:=\inf _{k \in \mathbb{N}} \alpha_{k}>0
$$

From (2.3) it immediately follows

$$
\left\|e^{t B}\right\|_{\mathcal{L}(H)} \leq e^{-\lambda t}, \quad t \geq 0 .
$$

Lemma 2.1. There exists $\gamma<1$ such that

$$
\sum_{k=1}^{\infty} e^{-t \alpha_{k}} \leq c(t \wedge 1)^{-\gamma} e^{-\lambda t}, \quad t \geq 0
$$

In particular

$$
\left\|e^{t B}\right\|_{2} \leq c(t \wedge 1)^{-\frac{\gamma}{2}} e^{-\lambda t}, \quad t \geq 0
$$

Proof. For any $\gamma>0$, there exists some $c_{\gamma}>0$ such that

$$
\sum_{k=1}^{\infty} e^{-\alpha_{k} t} \leq c_{\gamma} t^{-\gamma} \sum_{k=1}^{\infty} \alpha_{k}^{-\gamma}
$$

Now, for any second order uniformly elliptic operator on the interval $[0, L]$ having continuous coefficients, it holds $\alpha_{k} \sim k^{2}$. Hence, if we assume $\gamma>1 / 2$ and take $t \in(0,1]$, we have that (2.5) is satisfied. In the case $t>1$, thanks to (2.4) we have

$$
\sum_{k=1}^{\infty} e^{-\alpha_{k} t}=\sum_{k=1}^{\infty}\left|e^{(t-1) B} e^{B} e_{k}\right|_{H} \leq c e^{-(t-1) \lambda} \sum_{k=1}^{\infty}\left|e^{B} e_{k}\right|_{H} \leq c e^{-\lambda t},
$$

so that (2.5) follows in the general case.

\footnotetext{
${ }^{1}$ For any $s>0, W^{s, 2}(0, L)$ denotes the set of functions $x \in H$ such that

$$
[x]_{s, 2}:=\int_{[0, L]^{2}} \frac{|x(\xi)-x(\eta)|^{2}}{|\xi-\eta|^{2 s+1}} d \xi d \eta<\infty .
$$

$W^{s, 2}(0, L)$ is endowed with the norm $|x|_{s, 2}:=|x|_{H}+[x]_{s, 2}$.
} 
According to (2.6), there exists some $\delta>0$ such that

$$
\int_{0}^{t} s^{-\delta}\left\|e^{s B}\right\|_{2}^{2} d s<\infty, \quad t \geq 0
$$

As known (for a prof see e.g. [5]), this implies that the so-called stochastic convolution

$$
w^{B}(t):=\int_{0}^{t} e^{(t-s) B} d w(s), \quad t \geq 0,
$$

is a $p$ integrable $H$-valued process, for any $p \geq 1$, having continuous trajectories. Moreover, as a consequence of the dissipativity assumption (2.3), for any $p \geq 1$

$$
\sup _{t \geq 0} \mathbb{E}\left|w^{B}(t)\right|_{H}^{p}=: c_{p}<\infty .
$$

Concerning the reaction coefficient $f$ in the slow motion equation, we assume what follows.

Hypothesis 2. The mapping $f:[0, L] \times \mathbb{R}^{2} \rightarrow \mathbb{R}$ is measurable and $f(\xi, \cdot): \mathbb{R}^{2} \rightarrow \mathbb{R}$ is continuously differentiable, for almost all $\xi \in[0, L]$, with uniformly bounded derivatives.

Concerning the reaction coefficient $g$ in the fast motion equation, we assume the following conditions.

Hypothesis 3. 1. The mapping $g:[0, L] \times \mathbb{R}^{2} \rightarrow \mathbb{R}$ is measurable.

2. For each fixed $\sigma_{2} \in \mathbb{R}$ and almost all $\xi \in[0, L]$, the mapping $g\left(\xi, \cdot, \sigma_{2}\right): \mathbb{R} \rightarrow \mathbb{R}$ is of class $C^{1}$, with uniformly bounded derivatives.

3. For each fixed $\sigma_{1} \in \mathbb{R}$ and almost all $\xi \in[0, L]$, the mapping $g\left(\xi, \sigma_{1}, \cdot\right): \mathbb{R} \rightarrow \mathbb{R}$ is of class $C^{3}$, with uniformly bounded derivatives. Moreover,

$$
\sup _{\substack{\xi \in[0, L] \\ \sigma \in \mathbb{R}^{2}}}\left|\frac{\partial g}{\partial \sigma_{2}}(\xi, \sigma)\right|=: L_{g}<\lambda,
$$

where $\lambda$ is the positive constant introduced in (2.3).

In what follows we shall denote by $F$ and $G$ the Nemytskii operators associated respectively with $f$ and $g$, that is

$$
F(x, y)(\xi)=f(\xi, x(\xi), y(\xi)), \quad G(x, y)(\xi)=g(\xi, x(\xi), y(\xi)),
$$

for any $\xi \in[0, L]$ and $x, y \in H$. Due to the boundedness assumptions on their derivatives, functions $f$ and $g$ are Lipschitz-continuous and hence the mappings $F, G: H \times H \rightarrow H$ are Lipschitz-continuous.

Concerning their regularity properties, for any fixed $y \in H$ the mappings $F(\cdot, y)$ and $G(\cdot, y)$ are once Gâteaux differentiable in $H$ with

$$
D_{x} F(x, y) z=\frac{\partial f}{\partial \sigma_{1}}(\cdot, x, y) z, \quad D_{x} G(x, y) z=\frac{\partial g}{\partial \sigma_{1}}(\cdot, x, y) z
$$


Moreover, for any fixed $x \in H$, the mapping $F(x, \cdot): H \rightarrow H$ is once Gâteaux differentiable and the mapping $G(x, \cdot): H \rightarrow H$ is three times Gâteaux differentiable, with

$$
D_{y} F(x, y) z=\frac{\partial f}{\partial \sigma_{2}}(\cdot, x, y) z
$$

and

$$
D_{y}^{j} G(x, y)\left(z_{i}, \ldots, z_{j}\right)=\frac{\partial g^{j}}{\partial \sigma_{2}^{j}}(\cdot, x, y) z_{1} \cdots z_{j}, \quad j=1,2,3 .
$$

Notice that if $h \in L^{\infty}(0, L)$, then for any fixed $x, y \in H$ the mappings $\langle F(\cdot, y), h\rangle_{H}$ and $\langle F(x, \cdot), h\rangle_{H}$ are both Fréchet differentiable and

$$
\sup _{x, y \in H}\left|D\langle F(\cdot, y), h\rangle_{H}(x)\right|_{H} \leq L_{f}|h|_{H}, \quad \sup _{x, y \in H}\left|D\langle F(x, \cdot), h\rangle_{H}(y)\right|_{H} \leq L_{f}|h|_{H},
$$

where $L_{f}$ is the Lipschitz constant of $f$.

\section{Preliminary results on the fast motion equation}

As (2.7) holds and the mappings $F, G: H \times H \rightarrow H$ are both Lipschitz-continuous, for any $\epsilon>0$ and any initial conditions $x, y \in H$ system (1.7) admits a unique mild solution $\left(u^{\epsilon}, v^{\epsilon}\right) \in \mathcal{C}_{T, p} \times \mathcal{C}_{T, p}$, with $p \geq 1$ and $T>0$ (for a proof see e.g. [5, Theorem 7.6]). This means that there exist two unique processes $u^{\epsilon}$ and $v^{\epsilon}$, both in $\mathcal{C}_{T, p}$, such that for any $t \in[0, T]$

$$
u^{\epsilon}(t)=e^{t A} x+\int_{0}^{t} e^{(t-s) A} F\left(u^{\epsilon}(s), v^{\epsilon}(s)\right) d s
$$

and

$$
v^{\epsilon}(t)=e^{t B / \epsilon} y+\frac{1}{\epsilon} \int_{0}^{t} e^{(t-s) B / \epsilon} G\left(u^{\epsilon}(s), v^{\epsilon}(s)\right) d s+\frac{1}{\sqrt{\epsilon}} \int_{0}^{t} e^{(t-s) B / \epsilon} d w(s) .
$$

\subsection{The fast motion equation}

Now, for any fixed $x \in H$ we consider the problem

$$
\left\{\begin{array}{l}
\frac{\partial v}{\partial t}(t, \xi)=\mathcal{B} v(t, \xi)+g(\xi, x(\xi), v(t, \xi))+\frac{\partial w}{\partial t}(t, \xi), \quad t \geq 0, \quad \xi \in[0, L], \\
v(0, \xi)=y(\xi), \quad \xi \in[0, L], \quad \mathcal{N}_{2} v(t, \xi)=0, \quad t \geq 0, \quad \xi \in\{0, L\} .
\end{array}\right.
$$

By arguing as above, for any fixed slow component $x \in H$ and any initial datum $y \in H$, equation (3.2) admits a unique mild solution in $\mathcal{C}_{T, p}$, which will be denoted by $v^{x, y}(t)$.

Moreover, as proved for example in [4, Proposition 8.2.2], there exists $\theta>0$ such that for any $t_{0}>0$ and $p \geq 1$

$$
\sup _{t \geq t_{0}} \mathbb{E}\left|v^{x, y}(t)\right|_{C^{\theta}([0, L])}^{p}<\infty .
$$

Lemma 3.1. Under Hypotheses 1 and 3, for any $p \geq 1$ and $x, y \in H$

$$
\mathbb{E}\left|v^{x, y}(t)\right|_{H}^{p} \leq c_{p}\left(e^{-\delta p t}|y|_{H}^{p}+|x|_{H}^{p}+1\right), \quad t \geq 0,
$$

where $\delta:=\left(\lambda-L_{g}\right) / 2$. 
Proof. If we set $\rho(t):=v^{x, y}(t)-w^{B}(t)$, thanks to (2.4) and (2.9) and to the Lipschitzcontinuity of $G$, we have

$$
\begin{aligned}
& \frac{1}{2} \frac{d}{d t}|\rho(t)|_{H}^{2} \\
& =\langle B \rho(t), \rho(t)\rangle_{H}+\left\langle G\left(x, \rho(t)+w^{B}(t)\right)-G\left(x, w^{B}(t)\right), \rho(t)\right\rangle_{H}+\left\langle G\left(x, w^{B}(t)\right), \rho(t)\right\rangle_{H} \\
& \leq-\left(\lambda-L_{g}\right)|\rho(t)|_{H}^{2}+c\left(\left|w^{B}(t)\right|_{H}+|x|_{H}+1\right)|\rho(t)|_{H} \\
& \leq-\frac{\lambda-L_{g}}{2}|\rho(t)|_{H}^{2}+c\left(\left|w^{B}(t)\right|_{H}^{2}+|x|_{H}^{2}+1\right)
\end{aligned}
$$

and, by comparison, it easily follows

$$
\left|v^{x, y}(t)\right|_{H} \leq|\rho(t)|_{H}+\left|w^{B}(t)\right|_{H} \leq c\left(e^{-\frac{\lambda-L_{g}}{2} t}|y|_{H}+\sup _{s \geq 0}\left|w^{B}(s)\right|_{H}+|x|_{H}+1\right) .
$$

In particular, if we set $\delta:=\left(\lambda-L_{g}\right) / 2$, as a consequence of estimate (2.8) we obtain (3.4).

Since we are assuming that for each fixed $\sigma_{1} \in \mathbb{R}$ and almost all $\xi \in[0, L]$ the mapping $g\left(\xi, \sigma_{1}, \cdot\right): \mathbb{R} \rightarrow \mathbb{R}$ is of class $C^{3}$, with uniformly bounded derivatives, for any $T>0$ and $p \geq 1$ and for any fixed slow variable $x \in H$ the mapping

$$
y \in H \mapsto v^{x, y} \in \mathcal{H}_{T, p},
$$

is three times continuously differentiable (for a proof and all details see e.g. 4, Theorem 4.2.4]).

The first order derivative $D_{y} v^{x, y}(t) h$, at the point $y \in H$ and along the direction $h \in H$, is the solution of the first variation equation

$$
\left\{\begin{array}{l}
\frac{\partial z}{\partial t}(t, \xi)=\mathcal{B} z(t, \xi)+\frac{\partial g}{\partial \sigma_{2}}(\xi, x(\xi), y(\xi)) z(t, \xi), \\
z(0)=h, \quad \mathcal{N}_{2} z(t, \xi)=0, \quad \xi \in\{0, L\} .
\end{array}\right.
$$

Hence, thanks to (2.4) and (2.9) , it is immediate to check that for any $t \geq 0$

$$
\sup _{x, y \in H}\left|D_{y} v^{x, y}(t) h\right|_{H} \leq e^{-\delta t}|h|_{H}, \quad \mathbb{P}-\text { a.s. }
$$

where, as in the previous lemma, $\delta=\left(\lambda-L_{g}\right) / 2$. Moreover, as shown in [4, Lemma 4.2.2], for any $1 \leq r \leq p \leq \infty$ and $h \in L^{r}(0, L)$ we have that $D_{y} v^{x, y}(t) h \in L^{p}(0, L)$, $\mathbb{P}$-a.s. for $t>0$, and

$$
\sup _{y \in H}\left|D_{y} v^{x, y}(t) h\right|_{L^{p}} \leq \mu_{r, p}(t) t^{-\frac{p-r}{2 r p}}|h|_{L^{r}}, \quad \mathbb{P}-\text { a.s. }
$$

for a continuous increasing function $\mu_{r, p}$ which is independent of $x \in H$. 
Concerning the second and the third order derivatives, they are respectively solutions of the second and of the third variation equations. As proved in [4, Proposition 4.2.6], for any $h_{1}, h_{2}, h_{3} \in H$ and $p \geq 1$ both $D_{y}^{2} v^{x, y}(t)\left(h_{1}, h_{2}\right)$ and $D_{y}^{3} v^{x, y}(t)\left(h_{1}, h_{2}, h_{3}\right)$ belong to $L^{p}(0, L), \mathbb{P}$-a.s. for any $t \geq 0$, and

$$
\sup _{y \in H}\left|D_{y}^{j} v^{x, y}(t)\left(h_{1}, \ldots, h_{j}\right)\right|_{L^{p}} \leq \nu_{r, p}^{j}(t) \prod_{i=1}^{j}\left|h_{i}\right|_{H}, \quad \mathbb{P}-\text { a.s. },
$$

for $j=2,3$. It is important to notice that, as for $\mu_{r, p}$, due to the boundedness assumption on the derivatives of the reaction term $g$, all $\nu_{r, p}^{j}$ are continuous increasing functions independent of $x \in H$.

We conclude this subsection by proving the smooth dependence of the solution $v^{x, y}(t)$ of equation (3.2) on the frozen slow component $x \in H$. In the space $\mathcal{H}_{T, 2}$ we introduce the equivalent norm

$$
\left\||u \||:=\sup _{t \in[0, T]} e^{-\alpha t} \mathbb{E}|u(t)|_{H}^{2},\right.
$$

for some $\alpha>0$. Moreover, for any $x \in H$ and $v \in \mathcal{H}_{T, 2}$ we define

$$
\mathcal{F}(x, v)(t):=e^{t B} y+\int_{0}^{t} e^{(t-s) B} G(x, v(s)) d s+w^{B}(t), \quad t \in[0, T] .
$$

If $\alpha$ is chosen large enough, the mapping $\mathcal{F}(x, \cdot)$ is a contraction in the space $\mathcal{H}_{T, 2}$, endowed with the norm defined above.

It is easy to show that for all $v \in \mathcal{H}_{T, 2}$, the mapping $x \in H \mapsto \mathcal{F}(x, v) \in \mathcal{H}_{T, 2}$ is Fréchet differentiable and the derivative is continuous. Furthermore, for all $x \in H$ the mapping $v \in \mathcal{H}_{T, 2} \mapsto \mathcal{F}(x, v) \in \mathcal{H}_{T, 2}$ is Gâteaux differentiable and the derivative is continuous. Hence, by using the generalized theorem on contractions depending on a parameter given in [4, Proposition C.0.3], we have that the solution $v^{x, y}$ of equation (3.2), which is the fixed point of the mapping $\mathcal{F}(x, \cdot)$, is differentiable with respect to $x \in H$ and the derivative along the direction $h \in H$ satisfies the following equation

$$
\frac{d \rho}{d t}(t)=\left[B+G_{y}\left(x, v^{x, y}(t)\right)\right] \rho(t)+G_{x}\left(x, v^{x, y}(t)\right) h, \quad \rho(0)=0 .
$$

According to Hypothesis 3, we have

$$
\begin{aligned}
& \frac{1}{2} \frac{d}{d t}|\rho(t)|_{H}^{2}=\left\langle\left[B+G_{y}\left(x, v^{x, y}(t)\right)\right] \rho(t), \rho(t)\right\rangle_{H}+\left\langle G_{x}\left(x, v^{x, y}(t)\right) h, \rho(t)\right\rangle_{H} \\
& \leq-\frac{\lambda-L_{g}}{2}|\rho(t)|_{H}^{2}+\left|G_{x}\left(x, v^{x, y}(t)\right)\right|_{\mathcal{L}(H)}^{2}|h|_{H}^{2}
\end{aligned}
$$

so that, due to the boundedness of $G_{x}$,

$$
\sup _{x, y \in H}\left|D_{x} v^{x, y}(t) h\right|_{H} \leq c e^{-\frac{\lambda-L_{g}}{2} t}|h|_{H}, \quad \mathbb{P}-\text { a.s. }
$$




\subsection{The fast transition semigroup}

For any fixed $x \in H$, we denote by $P_{t}^{x}, t \geq 0$, the transition semigroup associated with the fast equation (3.2) with frozen slow component $x$. For any $\varphi \in B_{b}(H)$ and $t \geq 0$, it is defined by

$$
P_{t}^{x} \varphi(y)=\mathbb{E} \varphi\left(v^{x, y}(t)\right), \quad y \in H .
$$

As the mapping introduced in (3.6) is differentiable and (3.7) holds, it is immediate to check that $P_{t}^{x}$ is a Feller contraction semigroup and maps $C_{b}(H)$ into itself.

Thanks to estimate (3.4) and to (2.1), the semigroup $P_{t}^{x}$ is well defined on $\operatorname{Lip}(H)$ and for any $\varphi \in \operatorname{Lip}(H), x, y \in H$ and $t \geq 0$

$$
\left|P_{t}^{x} \varphi(y)\right| \leq[\varphi]_{\text {Lip }} \mathbb{E}\left|v^{x, y}(t)\right|_{H}+|\varphi(0)| \leq c[\varphi]_{\text {Lip }}\left(1+|x|_{H}+|y|_{H}\right)+|\varphi(0)| .
$$

Furthermore, $P_{t}^{x}$ maps $\operatorname{Lip}(H)$ into itself and according to (3.7)

$$
\left[P_{t}^{x} \varphi\right]_{\text {Lip }} \leq e^{-\delta t}[\varphi]_{\text {Lip }}, \quad t \geq 0 .
$$

As known, the semigroup $P_{t}^{x}$ is not strongly continuous on $C_{b}(H)$, in general. Nevertheless, it is weakly continuous on $C_{b}(H)$ (for a definition and all details we refer to [4, Appendix B]). For any $\lambda>0$ and $\varphi \in C_{b}(H)$, we set

$$
F^{x}(\lambda) \varphi(y):=\int_{0}^{\infty} e^{-\lambda t} P_{t}^{x} \varphi(y) d t, \quad x, y \in H
$$

As proved in [4, Proposition B.1.3 and Proposition B.1.4], since $P_{t}^{x}$ is a weakly continuous semigroup, for any $\lambda>0$ and $x \in H$ the linear operator $F^{x}(\lambda)$ is bounded from $C_{b}(H)$ into itself and there exists a unique closed linear operator $L^{x}: D(L) \subseteq C_{b}(H) \rightarrow C_{b}(H)$ such that

$$
F^{x}(\lambda)=R\left(\lambda, L^{x}\right) \quad \lambda>0 .
$$

Such an operator is, by definition, the infinitesimal weak generator of $P_{t}^{x}$.

It is important to stress that, thanks to (3.10) and (3.11), the operator $F^{x}(\lambda)$ is also well defined from $\operatorname{Lip}(H)$ into itself.

Concerning the regularity properties of $P_{t}^{x}$, as the mapping (3.6) is three times continuously differentiable, by differentiating under the sign of expectation, for any $t \geq 0$ and $k \leq 3$ we get

$$
\varphi \in \operatorname{Lip}^{k}(H) \Longrightarrow P_{t}^{x} \varphi \in \operatorname{Lip}^{k}(H),
$$

and thanks to estimates (3.7), for $k=1$, and (3.8), for $k=2,3$,

$$
\sup _{x \in H}\left[P_{t}^{x} \varphi\right]_{k} \leq c_{k}(t) \sum_{1 \leq h \leq k}[\varphi]_{h}, \quad t \geq 0
$$

where $c_{k}(t)$ is some continuous increasing function. Moreover, the semigroup $P_{t}^{x}$ has a smoothing effect. Actually, as proved in [4, Theorem 4.4.5], for any $t>0$

$$
\varphi \in B_{b}(H) \Longrightarrow P_{t}^{x} \varphi \in C_{b}^{3}(H)
$$


and for any $0 \leq i \leq j \leq 3$

$$
\sup _{x \in H}\left\|D^{j}\left(P_{t}^{x} \varphi\right)\right\|_{0} \leq c(t \wedge 1)^{-\frac{j-i}{2}}\|\varphi\|_{i}, \quad t>0 .
$$

By adapting the arguments used in [4, Theorem 4.4.5], it is possible to prove that if $\varphi \in \operatorname{Lip}(H)$, then $P_{t}^{x} \varphi$ is three times continuously differentiable, for any $t>0$. Moreover, the following estimates for the derivatives of $P_{t}^{x} \varphi$ hold

$$
\left[P_{t}^{x} \varphi\right]_{1}=\sup _{y \in H}\left|D P_{t}^{x} \varphi(y)\right|_{H} \leq e^{-\delta t}[\varphi]_{\mathrm{Lip}}
$$

and for $j=2,3$

$$
\left|D^{j} P_{t}^{x} \varphi(y)\right|_{\mathcal{L}^{j}(H)} \leq c(t \wedge 1)^{-\frac{j-1}{2}}\left([\varphi]_{\text {Lip }}\left(1+|x|_{H}+|y|_{H}\right)+|\varphi(0)|\right) .
$$

Moreover, by adapting the proof of [4, Theorem 5.2.4], which is given for bounded functions, to the case of general Lipschitz-continuous functions, it is possible to prove the following crucial fact.

Theorem 3.2. Under Hypotheses 1 and 3 , the operator $D^{2}\left(P_{t}^{x} \varphi\right)(y)$ belongs to $\mathcal{L}_{1}(H)$, for any fixed $x, y \in H, t>0$ and $\varphi \in \operatorname{Lip}(H)$. Besides, the mapping

$$
(t, y) \in(0, \infty) \times H \mapsto \operatorname{Tr}\left[D^{2}\left(P_{t}^{x} \varphi\right)(y)\right] \in \mathbb{R}
$$

is continuous and

$$
\left|\operatorname{Tr}\left[D^{2}\left(P_{t}^{x} \varphi\right)(y)\right]\right| \leq c_{\gamma}(t \wedge 1)^{-\frac{1+\gamma}{2}}\left([\varphi]_{\text {Lip }}\left(1+|x|_{H}+|y|_{H}\right)+|\varphi(0)|\right),
$$

where $\gamma$ is the constant introduced in (2.5).

Remark 3.3. Even if the semigroup $P_{t}^{x}$ has a smoothing effect, the proof of the validity of the trace-class property for the operator $D^{2}\left(P_{t}^{x} \varphi\right)(y)$ is far from being trivial. Actually, it is based on the two following facts. First (see [4, Lemma 5.2.1]), if $\left\{e_{k}\right\}_{k \in \mathbb{N}}$ is the orthonormal basis introduced in Hypothesis 1 and if $\gamma<1$ is the constant introduced in (2.5), then it holds

$$
\sup _{y \in H} \sum_{k=1}^{\infty} \int_{0}^{t}\left|D_{y} v^{x, y}(s) e_{k}\right|_{H}^{2} d s \leq c(t) t^{1-\gamma}, \quad \mathbb{P}-\text { a.s. }
$$

for some continuous increasing function $c(t)$ independent of $x \in H$. Secondly (see [4, Lemma 5.2.2]), there exists some continuous increasing function $c(t)$ such that for any $N \in \mathcal{L}(H)$ and $x \in H$

$$
\sup _{y \in H} \sum_{k=1}^{\infty} \int_{0}^{t}\left|D_{y}^{2} v^{x, y}(s)\left(e_{k}, N e_{k}\right)\right|_{H}^{2} d s \leq c(t)\|N\|, \quad \mathbb{P}-\text { a.s. }
$$

It is important to stress that both the estimate for the first derivative and the estimate for the second derivative are a consequence of (2.6) and (2.5). 


\subsection{The asymptotic behavior of the fast equation}

We describe here the asymptotic behavior of the semigroup $P_{t}^{x}$. Namely, we show that, for any fixed $x \in H$, it admits a unique invariant measure $\mu^{x}$ which is explicitly given and we describe the convergence of $P_{t}^{x}$ to equilibrium. Most of these results are basically known in the literature, but we shortly recall them for the reader convenience.

According to (2.6), the self-adjoint operator

$$
\int_{0}^{\infty} e^{2 s B} d s=\frac{1}{2}(-B)^{-1}
$$

is well defined in $\mathcal{L}_{1}(H)$, so that the Gaussian measure $\mathcal{N}\left(0,(-B)^{-1} / 2\right)$ of zero mean and covariance operator $(-B)^{-1} / 2$ is well defined on $(H, \mathcal{B}(H))$.

For any $x, y \in H$ we define

$$
U(x, y):=\int_{0}^{1}\langle G(x, \theta y), y\rangle_{H} d \theta=\int_{0}^{L} \int_{0}^{y(\xi)} g(\xi, x(\xi), s) d s d \xi .
$$

Due to the Lipschitz-continuity of $g(\xi, \cdot)$ (see Hypothesis 3), for any $x, y \in H$ we have

$$
|G(x, y)|_{H} \leq L_{g}|y|_{H}+c|x|_{H}+|G(0,0)|_{H},
$$

so that for any $\eta>0$ we can fix a constant $c_{\eta} \geq 0$ such that

$$
|U(x, y)| \leq \frac{L_{g}+\eta}{2}|y|_{H}^{2}+c_{\eta}\left(1+|x|_{H}^{2}\right) .
$$

As $\eta>0$ can be chosen as small as we wish, thanks to (2.9) this implies that for any fixed $x \in H$ the mapping

$$
y \in H \mapsto \exp 2 U(x, y) \in \mathbb{R}
$$

is integrable with respect to the Gaussian measure $\mathcal{N}\left(0,(-B)^{-1} / 2\right)$ and

$$
Z(x):=\int_{H} \exp 2 U(x, y) \mathcal{N}\left(0,(-B)^{-1} / 2\right) d y \in(0, \infty), \quad x \in H .
$$

This means that for each fixed $x \in H$ the measure

$$
\mu^{x}(d y):=\frac{1}{Z(x)} \exp 2 U(x, y) \mathcal{N}\left(0,(-B)^{-1} / 2\right)(d y)
$$

is well defined on $(H, \mathcal{B}(H))$.

Now, it is immediate to check that the mapping $U(x, \cdot): H \rightarrow \mathbb{R}$ is differentiable and

$$
U_{y}(x, y)=G(x, y), \quad x, y \in H .
$$

Therefore, as well known from the existing literature, the measure $\mu^{x}$ defined in (3.17) is invariant for equation (3.2).

Because of the way the measure $\mu^{x}$ has been constructed, we immediately have that it has all moments finite. In particular, for any $x \in H$ we have

$$
\operatorname{Lip}(H) \subset L^{p}\left(H, \mu^{x}\right), \quad p \geq 1 .
$$

In the next lemma we show how the moments of $\mu^{x}$ can be estimated in terms of the slow variable $x$. 
Lemma 3.4. Under Hypotheses 1 and 3, for any $x \in H$ and $p \geq 1$

$$
\int_{H}|z|_{H}^{p} \mu^{x}(d z) \leq c\left(1+|x|_{H}^{p}\right) .
$$

Proof. By using the invariance of $\mu^{x}$, thanks to estimate (3.4) for any $p \geq 1$ and $t \geq 0$ we have

$$
\begin{aligned}
& \int_{H}|z|_{H}^{p} \mu^{x}(d z)=\int_{H} P_{t}^{x}|z|_{H}^{p} \mu^{x}(d z)=\int_{H} \mathbb{E}\left|v^{x, z}(t)\right|_{H}^{p} \mu^{x}(d z) \\
& \leq c e^{-\delta p t} \int_{H}|z|_{H}^{p} \mu^{x}(d z)+c\left(1+|x|_{H}^{p}\right)
\end{aligned}
$$

Then, if we take $t=t_{0}$ such that $c e^{-\delta p t_{0}}<1$, we have (3.20).

Once we have the explicit invariant measure $\mu^{x}$, we show that it is unique and we describe its convergence to equilibrium.

Theorem 3.5. Under Hypotheses 1 and 3, for any fixed $x \in H$ equation (3.2) admits a unique ergodic invariant measure $\mu^{x}$, which is strongly mixing and such that for any $\varphi \in B_{b}(H)$ and $x, y \in H$

$$
\left|P_{t}^{x} \varphi(y)-\int_{H} \varphi(z) \mu^{x}(d z)\right| \leq c\left(1+|x|_{H}+|y|_{H}\right) e^{-\delta t}(t \wedge 1)^{-\frac{1}{2}}\|\varphi\|_{0},
$$

where $\delta:=\left(\lambda-L_{g}\right) / 2$.

Proof. We fix $y, z \in H$ and set $\rho(t):=v^{x, y}(t)-v^{x, z}(t)$. We have

$$
\frac{1}{2} \frac{d}{d t}|\rho(t)|_{H}^{2}=\langle B \rho(t), \rho(t)\rangle_{H}+\left\langle G\left(x, v^{x, y}(t)\right)-G\left(x, v^{x, z}(t)\right), \rho(t)\right\rangle_{H},
$$

and then, according to (2.4) and (2.9), we easily get

$$
\left|v^{x, y}(t)-v^{x, z}(t)\right|_{H}^{2}=|\rho(t)|_{H}^{2} \leq e^{-2\left(\lambda-L_{g}\right) t}|y-z|_{H}^{2}, \quad \mathbb{P}-\text { a.s. }
$$

This means that for any $\varphi \in \operatorname{Lip}(H)$

$$
\left|P_{t}^{x} \varphi(y)-P_{t}^{x} \varphi(z)\right| \leq[\varphi]_{\text {Lip }} \mathbb{E}\left|v^{x, y}(t)-v^{x, z}(t)\right|_{H} \leq[\varphi]_{\text {Lip }} e^{-\left(\lambda-L_{g}\right) t}|y-z|_{H}, \quad t \geq 0 .
$$

Hence, if $\varphi \in B_{b}(H)$, due to the semigroup law and to estimate (3.12) (with $j=1$ and $i=0$ ), for any $t>0$ we obtain

$$
\left|P_{t}^{x} \varphi(y)-P_{t}^{x} \varphi(z)\right| \leq\left[P_{t / 2}^{x} \varphi\right]_{1} e^{-\delta t}|y-z|_{H} \leq c\|\varphi\|_{0}(t \wedge 1)^{-\frac{1}{2}} e^{-\delta t}|y-z|_{H},
$$

where $\delta:=\left(\lambda-L_{g}\right) / 2$. In particular,

$$
\lim _{t \rightarrow \infty} P_{t}^{x} \varphi(y)-P_{t}^{x} \varphi(z)=0
$$

so that the invariant measure $\mu^{x}$ is unique and strongly mixing. 
Now, due to the invariance of $\mu^{x}$, if $\varphi \in B_{b}(H)$ from (3.22) we have

$$
\begin{aligned}
& \left|P_{t}^{x} \varphi(y)-\int_{H} \varphi(z) \mu^{x}(d z)\right|=\left|\int_{H}\left[P_{t}^{x} \varphi(y)-P_{t}^{x} \varphi(z)\right] \mu^{x}(d z)\right| \\
& \leq c\|\varphi\|_{0} e^{-\delta t}(t \wedge 1)^{-\frac{1}{2}} \int_{H}|y-z|_{H} \mu^{x}(d z) \\
& \leq c\|\varphi\|_{0} e^{-\delta t}(t \wedge 1)^{-\frac{1}{2}}\left(|y|_{H}+\int_{H}|z|_{H} \mu^{x}(d z)\right)
\end{aligned}
$$

and then, thanks to (3.20) (with $p=1$ ), we obtain (3.21).

Remark 3.6. From the proof of estimate (3.21), we immediately see that if $\varphi \in \operatorname{Lip}(H)$, then for any $x, y \in H$

$$
\left|P_{t}^{x} \varphi(y)-\int_{H} \varphi(z) \mu^{x}(d z)\right| \leq c\left(1+|x|_{H}+|y|_{H}\right) e^{-\delta t}[\varphi]_{\mathrm{Lip}},
$$

where $\delta:=\left(\lambda-L_{g}\right) / 2$.

\subsection{The Kolmogorov equation associated with the fast equation}

For any frozen slow component $x \in H$, the Kolmogorov operator associated with equation (3.2) is given by the following second order differential operator

$$
\mathcal{L}^{x} \varphi(y)=\frac{1}{2} \operatorname{Tr}\left[D^{2} \varphi(y)\right]+\langle B y+G(x, y), D \varphi(y)\rangle_{H}, \quad y \in D(B) .
$$

The operator $\mathcal{L}^{x}$ is defined for functions $\varphi: H \rightarrow \mathbb{R}$ which are twice continuously differentiable, such that the operator $D^{2} \varphi(y)$ is in $\mathcal{L}_{1}(H)$, for all $y \in H$, and the mapping

$$
y \in H \mapsto \operatorname{Tr} D^{2} \varphi(y) \in \mathbb{R},
$$

is continuous. In what follows it will be important to study the solvability of the elliptic equation associated with the infinite dimensional operator $\mathcal{L}^{x}$

$$
\lambda \varphi(y)-\mathcal{L}^{x} \varphi(y)=\psi(y), \quad y \in D(B),
$$

for any fixed $x \in H, \lambda>0$ and $\psi: H \rightarrow \mathbb{R}$ regular enough. To this purpose we recall the notion of strict solution for the elliptic problem (3.24).

Definition 3.7. A function $\varphi$ is a strict solution of problem (3.24) if

1. $\varphi$ belongs to $D\left(\mathcal{L}^{x}\right)$, that is $\varphi: H \rightarrow \mathbb{R}$ is twice continuously differentiable, the operator $D^{2} \varphi(y) \in \mathcal{L}_{1}(H)$, for any $y \in H$, and the mapping $y \mapsto \operatorname{Tr} D^{2} \varphi(y)$ is continuous on $H$ with values in $\mathbb{R}$;

2. $\varphi(y)$ satisfies (3.24), for any $y \in D(B)$. 
In the next theorem we see how it is possible to get the existence of a strict solution of problem (3.24) in terms of the Laplace transform of the semigroup $P_{t}^{x}$ (see subsection 3.2 for the definition and [4, Theorem 5.4.3] for the proof).

Theorem 3.8. Fix any $x \in H$ and $\lambda>0$. Then under Hypotheses 1 and 3 , for any $\psi \in \operatorname{Lip}(H)$ the function

$$
y \in H \mapsto \varphi(x, y):=\int_{0}^{\infty} e^{-\lambda t} P_{t}^{x} \psi(y) d t \in \mathbb{R}
$$

is a strict solution of problem (3.24).

Remark 3.9. A detailed proof of the theorem above can be found in [4, Theorem 5.4.3] in the case $\psi \in C_{b}^{1}(H)$. The case of $\psi \in \operatorname{Lip}(H)$ is analogous: we have to prove that for any $\psi \in \operatorname{Lip}(H)$ the function $R\left(\lambda, L^{x}\right) \psi$ is a strict solution. To this purpose, by using (3.13) and (3.14), we have that $R\left(\lambda, L^{x}\right) \psi$ is twice continuously differentiable and then, thanks to Theorem 3.2 and estimate (3.15), we have that $D^{2}\left[R\left(\lambda, L^{x}\right) \psi\right] \in \mathcal{L}_{1}(H)$ and continuity for the trace holds. Notice that in all these results it is crucial that $\psi \in \operatorname{Lip}(H)$, because in this case all singularities arising at $t=0$ are integrable.

\section{A priori bounds for the solution of the system}

With the notations introduced in section 2, system (1.7) can be written as

$$
\left\{\begin{array}{l}
\frac{d u^{\epsilon}}{d t}(t)=A u^{\epsilon}(t)+F\left(u^{\epsilon}(t), v^{\epsilon}(t)\right), \quad u^{\epsilon}(0)=x \\
d v^{\epsilon}(t)=\frac{1}{\epsilon}\left[B v^{\epsilon}(t)+G\left(u^{\epsilon}(t), v^{\epsilon}(t)\right)\right] d t+\frac{1}{\sqrt{\epsilon}} d w(t), \quad v^{\epsilon}(0)=y .
\end{array}\right.
$$

Our aim here is proving uniform bounds with respect to $\epsilon>0$ for the solutions $u^{\epsilon}$ and $v^{\epsilon}$ of system (4.1).

Lemma 4.1. Under Hypotheses 1, 2 and 3, for any $x, y \in H$ and $T>0$ we have

$$
\sup _{\epsilon>0} \mathbb{E} \sup _{t \in[0, T]}\left|u^{\epsilon}(t)\right|_{H}^{2} \leq c_{T}\left(1+|x|_{H}^{2}+|y|_{H}^{2}\right)
$$

and

$$
\sup _{\epsilon>0} \sup _{t \in[0, T]} \mathbb{E}\left|v^{\epsilon}(t)\right|_{H}^{2} \leq c_{T}\left(1+|x|_{H}^{2}+|y|_{H}^{2}\right)
$$

for some constant $c_{T}>0$.

Proof. We have

$$
\begin{aligned}
& \frac{1}{2} \frac{d}{d t}\left|u^{\epsilon}(t)\right|_{H}^{2}=\left\langle A u^{\epsilon}(t), u^{\epsilon}(t)\right\rangle_{H}+\left\langle F\left(u^{\epsilon}(t), v^{\epsilon}(t)\right)-F\left(0, v^{\epsilon}(t)\right), u^{\epsilon}(t)\right\rangle_{H} \\
& +\left\langle F\left(0, v^{\epsilon}(t)\right), u^{\epsilon}(t)\right\rangle_{H} \leq c\left|u^{\epsilon}(t)\right|_{H}^{2}+c\left(1+\left|v^{\epsilon}(t)\right|_{H}^{2}\right),
\end{aligned}
$$


so that

$$
\left|u^{\epsilon}(t)\right|_{H}^{2} \leq e^{c t}|x|_{H}^{2}+c \int_{0}^{t} e^{c(t-s)}\left(1+\left|v^{\epsilon}(s)\right|_{H}^{2}\right) d s .
$$

Now, for any $\epsilon>0$ we denote by $w^{\epsilon, B}(t)$ the solution of the problem

$$
d z(t)=\frac{1}{\epsilon} B z(t) d t+\frac{1}{\sqrt{\epsilon}} d w(t), \quad z(0)=0
$$

We have

$$
w^{\epsilon, B}(t)=\frac{1}{\sqrt{\epsilon}} \int_{0}^{t} e^{(t-s) B / \epsilon} d w(s),
$$

and, due to (2.6), with a simple change of variables we get

$$
\mathbb{E}\left|w^{\epsilon, B}(t)\right|_{H}^{2}=\frac{1}{\epsilon} \int_{0}^{t}\left\|e^{(t-s) B / \epsilon}\right\|_{2}^{2} d s=\int_{0}^{t / \epsilon}\left\|e^{\rho B}\right\|_{2}^{2} d \rho \leq c \int_{0}^{\infty}(\rho \wedge 1)^{-\gamma} e^{-2 \lambda \rho} d \rho<\infty .
$$

This means that

$$
\sup _{\epsilon>0} \sup _{t \geq 0} \mathbb{E}\left|w^{\epsilon, B}(t)\right|_{H}^{2}<\infty .
$$

Notice that the same uniform bound is true for moments of any order of the $H$-norm of $w^{\epsilon, B}(t)$.

If we set $\rho^{\epsilon}(t):=v^{\epsilon}(t)-w^{\epsilon, B}(t)$, by proceeding as in the proof of Lemma 3.1 we have

$$
\frac{1}{2} \frac{d}{d t}\left|\rho^{\epsilon}(t)\right|_{H}^{2} \leq-\frac{\lambda-L_{g}}{2 \epsilon}\left|\rho^{\epsilon}(t)\right|_{H}^{2}+\frac{c}{\epsilon}\left(1+\left|u^{\epsilon}(t)\right|_{H}^{2}+\left|w^{\epsilon, B}(t)\right|_{H}^{2}\right) .
$$

Hence, by comparison

$$
\left|\rho^{\epsilon}(t)\right|_{H}^{2} \leq e^{-\frac{\lambda-L_{g}}{\epsilon} t}|y|_{H}^{2}+\frac{c}{\epsilon} \int_{0}^{t} e^{-\frac{\lambda-L_{g}}{\epsilon}(t-s)}\left(1+\left|u^{\epsilon}(s)\right|_{H}^{2}+\left|w^{\epsilon, B}(s)\right|_{H}^{2}\right) d s .
$$

According to (4.4), this implies

$$
\begin{aligned}
& \left|v^{\epsilon}(t)\right|_{H}^{2} \leq 2\left|w^{\epsilon, B}(t)\right|_{H}^{2}+2\left|\rho^{\epsilon}(t)\right|_{H}^{2} \leq 2\left|w^{\epsilon, B}(t)\right|_{H}^{2}+c_{T}\left(1+|x|_{H}^{2}+|y|_{H}^{2}\right) \\
& +\frac{c_{T}}{\epsilon} \int_{0}^{t} e^{-\frac{\lambda-L_{g}}{\epsilon}}(t-s) \int_{0}^{s}\left|v^{\epsilon}(r)\right|_{H}^{2} d r d s+\frac{c}{\epsilon} \int_{0}^{t} e^{-\frac{\lambda-L_{g}}{\epsilon}(t-s)}\left|w^{\epsilon, B}(s)\right|_{H}^{2} d s
\end{aligned}
$$

and by taking expectation, thanks to (4.5) we have

$$
\begin{aligned}
& \mathbb{E}\left|v^{\epsilon}(t)\right|_{H}^{2} \leq c_{T}\left(1+|x|_{H}^{2}+|y|_{H}^{2}\right) \\
& +\frac{c_{T}}{\epsilon} \int_{0}^{t} e^{-\frac{\lambda-L_{g}}{\epsilon}(t-s)} \int_{0}^{s} \mathbb{E}\left|v^{\epsilon}(r)\right|_{H}^{2} d r d s+\frac{c}{\epsilon} \int_{0}^{t} e^{-\frac{\lambda-L_{g}}{\epsilon} s} d s .
\end{aligned}
$$

With a change of variables, this yields

$$
\begin{aligned}
& \mathbb{E}\left|v^{\epsilon}(t)\right|_{H}^{2} \leq c_{T}\left(1+|x|_{H}^{2}+|y|_{H}^{2}\right) \\
& +c_{T} \int_{0}^{t}\left[\int_{0}^{\frac{t-r}{\epsilon}} e^{-\left(\lambda-L_{g}\right) \sigma} d \sigma\right] \mathbb{E}\left|v^{\epsilon}(r)\right|_{H}^{2} d r+c \int_{0}^{\frac{t}{\epsilon}} e^{-\left(\lambda-L_{g}\right) s} d s \\
& \leq c_{T}\left(1+|x|_{H}^{2}+|y|_{H}^{2}\right)+c_{T} \int_{0}^{t} \mathbb{E}\left|v^{\epsilon}(r)\right|_{H}^{2} d r,
\end{aligned}
$$


so that

$$
\sup _{t \in[0, T]} \mathbb{E}\left|v^{\epsilon}(t)\right|_{H}^{2} \leq c_{T}\left(1+|x|_{H}^{2}+|y|_{H}^{2}\right),
$$

which gives (4.3). By replacing the estimate above in (4.4), we immediately obtain (4.2).

Remark 4.2. In the previous lemma we have proved uniform bounds, with respect to $\epsilon>0$, for $\sup _{t \in[0, T]} \mathbb{E}\left|v^{\epsilon}(t)\right|_{H}$ and not for $\mathbb{E} \sup _{t \in[0, T]}\left|v^{\epsilon}(t)\right|_{H}$. This is a consequence of the fact that we can only prove the following estimate for the second moment of the $C([0, T] ; H)$-norm of the stochastic convolution $w^{\epsilon, B}$

$$
\mathbb{E} \sup _{t \in[0, T]}\left|w^{\epsilon, B}(t)\right|_{H}^{2} \leq c_{T, \delta} \epsilon^{\delta-1}, \quad t \in[0, T]
$$

for any $0<\delta<1 / 2$. Then, due to the previous estimate, we are only able to prove that

$$
\mathbb{E} \sup _{t \in[0, T]}\left|v^{\epsilon}(t)\right|_{H}^{2} \leq c_{T, \delta}\left(1+|x|_{H}^{2}+|y|_{H}^{2}+\epsilon^{\delta-1}\right), \quad \epsilon>0 .
$$

Theorem 4.3. Assume that $x \in W^{\alpha, 2}(0, L)$, for some $\alpha>0$. Then, under Hypotheses 1, [ and [3, for any $T>0$ the family of probability measures $\left\{\mathcal{L}\left(u^{\epsilon}\right)\right\}_{\epsilon>0}$ is tight in $C((0, T] ; H) \cap$ $L^{\infty}(0, T ; H)$.

Proof. As known, if $\delta \leq 1 / 4$

$$
W^{2 \delta, 2}(0, L)=\left(H, W_{\mathcal{N}_{i}}^{2,2}(0, L)\right)_{\delta, \infty}=\left\{x \in H: \sup _{t \in(0,1]} t^{-\delta}\left|e^{t A} x-x\right|_{H}<\infty\right\},
$$

with equivalence of norms. Moreover, if $f \in L^{2}(0, T ; H)$, it is possible to prove that for any $t>s$ and $\delta<1 / 2$

$$
\left|\int_{0}^{t} e^{(t-r) A} f(r) d r-\int_{0}^{s} e^{(s-r) A} f(r) d r\right|_{H} \leq c_{T, \delta}(t-s)^{\delta}\|f\|_{L^{2}(0, T ; H)} .
$$

Then, if $x \in W^{\alpha, 2}(0, L)$, for any $t>s$ and $\theta \leq 1 / 4 \wedge \alpha / 2$ we have

$$
\begin{aligned}
& \left|u^{\epsilon}(t)-u^{\epsilon}(s)\right|_{H} \\
& \leq\left|e^{s A}\left(e^{(t-s) A} x-x\right)\right|_{H}+\left|\int_{0}^{t} e^{(t-r) A} F\left(u^{\epsilon}(r), v^{\epsilon}(r)\right) d r-\int_{0}^{s} e^{(s-r) A} F\left(u^{\epsilon}(r), v^{\epsilon}(r)\right) d r\right|_{H} \\
& \leq c_{T, \theta}(t-s)^{\theta}|x|_{2 \theta, 2}+c_{T, \theta}(t-s)^{\theta}\left\|F\left(u^{\epsilon}, v^{\epsilon}\right)\right\|_{L^{2}(0, T ; H)} .
\end{aligned}
$$

This implies that for any $\theta \leq 1 / 4 \wedge \alpha / 2$

$$
\left[u^{\epsilon}\right]_{C^{\theta}([0, T] ; H)}=\sup _{s \neq t} \frac{\left|u^{\epsilon}(t)-u^{\epsilon}(s)\right|}{|t-s|^{\theta}} \leq c_{T}\left(|x|_{2 \theta, 2}+\left\|F\left(u^{\epsilon}, v^{\epsilon}\right)\right\|_{L^{2}(0, T ; H)}\right) .
$$

Now, according to estimates (4.2) and (4.3), we have

$$
\mathbb{E}\left\|F\left(u^{\epsilon}, v^{\epsilon}\right)\right\|_{L^{2}(0, T ; H)} \leq c\left(1+\mathbb{E}\left|u^{\epsilon}\right|_{L^{2}([0, T] ; H)}+\mathbb{E}\left|v^{\epsilon}\right|_{L^{2}([0, T] ; H)}\right) \leq c_{T}\left(1+|x|_{H}+|y|_{H}\right),
$$


and hence

$$
\sup _{\epsilon>0} \mathbb{E}\left|u^{\epsilon}\right|_{C^{\theta}([0, T] ; H)} \leq c_{T}\left(1+|x|_{2 \theta, 2}+|y|_{H}\right) .
$$

Next, if $\theta<1 / 2 \wedge \alpha$, thanks to (2.2) for any $t \in[0, T]$ we have

$$
\begin{aligned}
& \left|u^{\epsilon}(t)\right|_{\theta, 2} \leq c_{T}|x|_{\theta, 2}+c_{T} \int_{0}^{t}(t-s)^{-\frac{\theta}{2}}\left|F\left(u^{\epsilon}(s), v^{\epsilon}(s)\right)\right|_{H} d s \\
& \leq c_{T}|x|_{\theta, 2}+c_{T, \theta}\left\|F\left(u^{\epsilon}, v^{\epsilon}\right)\right\|_{L^{2}(0, T ; H)} .
\end{aligned}
$$

Then, by using again (4.10), we have

$$
\sup _{\epsilon>0} \mathbb{E} \sup _{t \in[0, T]}\left|u^{\epsilon}(t)\right|_{\theta, 2} \leq c_{T, \theta}\left(1+|x|_{\theta, 2}+|y|_{H}\right) .
$$

Combining together (4.11) and (4.12), we conclude that for any $\eta>0$ there exists $R(\eta)>0$ such that

$$
\mathbb{P}\left(u^{\epsilon} \in \mathcal{K}_{R(\eta)}\right) \geq 1-\eta, \quad \epsilon>0
$$

where, by the Ascoli-Arzelà theorem, $\mathcal{K}_{R(\eta)}$ is the compact subset of $C((0, T] ; H) \cap L^{\infty}(0, T ; H)$ defined by

$$
\mathcal{K}_{R(\eta)}:=\left\{u: \sup _{t \in[0, T]}|u(t)|_{\theta, 2} \leq R(\eta),|u|_{C^{\theta}([0, T] ; H)} \leq R(\eta)\right\},
$$

for some $\theta<1 / 4 \wedge \alpha / 2$. This implies the tightness of the family $\left\{\mathcal{L}\left(u^{\epsilon}\right)\right\}_{\epsilon>0}$ in $C((0, T] ; H) \cap$ $L^{\infty}(0, T ; H)$.

We conclude the present section by proving that if $x$ and $y$ are taken in $W^{\alpha, 2}(0, L)$, for some $\alpha>0$, then $u^{\epsilon}(t) \in D(A)$, for $t>0$. Moreover, we provide an estimate for the momentum of the norm of $A u^{\epsilon}(t)$, which is uniform with respect to $\epsilon \in(0,1]$.

Lemma 4.4. Assume that $x, y \in W^{\alpha, 2}(0, L)$, for some $\alpha \in(0,2]$. Then, under Hypotheses 1, 2 and [, we have that $u^{\epsilon}(t) \in D(A), \mathbb{P}$-a.s., for any $t>0$ and $\epsilon>0$. Moreover, for any $T>0$ and $\epsilon \in(0,1]$ it holds

$$
\mathbb{E}\left|A u^{\epsilon}(t)\right|_{H} \leq c t^{\frac{\alpha}{2}-1}|x|_{\alpha, 2}+c_{T}\left(1+\epsilon^{-\frac{\alpha \vee(1-\gamma)}{2}}\right)\left(1+|x|_{\alpha, 2}+|y|_{\alpha, 2}\right), \quad t \in(0, T],
$$

where $\gamma$ is the constant introduced in (2.5).

Proof. We decompose $u^{\epsilon}(t)$ as

$$
\begin{aligned}
& u^{\epsilon}(t)=u_{1}^{\epsilon}(t)+u_{2}^{\epsilon}(t):=\left[e^{t A} x+\int_{0}^{t} e^{(t-s) A} F\left(u^{\epsilon}(t), v^{\epsilon}(t)\right) d s\right] \\
& +\int_{0}^{t} e^{(t-s) A}\left[F\left(u^{\epsilon}(s), v^{\epsilon}(s)\right)-F\left(u^{\epsilon}(t), v^{\epsilon}(t)\right)\right] d s .
\end{aligned}
$$


According to (2.2) we have

$$
\begin{aligned}
& \left|A u_{1}^{\epsilon}(t)\right|_{H} \leq\left|A e^{t A} x\right|_{H}+\left|\left(e^{t A}-I\right) F\left(u^{\epsilon}(t), v^{\epsilon}(t)\right)\right|_{H} \\
& \leq c_{T} t^{\frac{\alpha}{2}-1}|x|_{\alpha, 2}+c_{T}\left(1+\left|u^{\epsilon}(t)\right|_{H}+\left|v^{\epsilon}(t)\right|_{H}\right),
\end{aligned}
$$

so that, thanks to (4.2) and (4.3)

$$
\mathbb{E}\left|A u_{1}^{\epsilon}(t)\right|_{H} \leq c_{T} t^{\frac{\alpha}{2}-1}|x|_{\alpha, 2}+c_{T}\left(1+|x|_{H}+|y|_{H}\right), \quad t \in(0, T] .
$$

Concerning $u_{2}^{\epsilon}(t)$, we have

$$
\begin{aligned}
& \left|A u_{2}^{\epsilon}(t)\right|_{H} \leq c_{T} \int_{0}^{t}(t-s)^{-1}\left(\left|u^{\epsilon}(t)-u^{\epsilon}(s)\right|_{H}+\left|v^{\epsilon}(t)-v^{\epsilon}(s)\right|_{H}\right) d s \\
& \leq c_{T} \int_{0}^{t}(t-s)^{\frac{\alpha}{2}-1} d s\left[u^{\epsilon}\right]_{C^{\frac{\alpha}{2}}(0, T ; H)}+c \int_{0}^{t}(t-s)^{-1}\left|v^{\epsilon}(t)-v^{\epsilon}(s)\right|_{H} d s,
\end{aligned}
$$

and then, due to (4.11), by taking expectation we have

$$
\mathbb{E}\left|A u_{2}^{\epsilon}(t)\right|_{H} \leq c_{T}\left(1+|x|_{\alpha, 2}+|y|_{H}\right)+c \int_{0}^{t}(t-s)^{-1} \mathbb{E}\left|v^{\epsilon}(t)-v^{\epsilon}(s)\right|_{H} d s .
$$

This means that, in order to conclude the proof, we have to estimate $\mathbb{E}\left|v^{\epsilon}(t)-v^{\epsilon}(s)\right|_{H}$, for any $0 \leq s<t \leq T$.

It holds

$$
\begin{aligned}
& v^{\epsilon}(t)-v^{\epsilon}(s)=\left[e^{t \frac{B}{\epsilon}} y-e^{s \frac{B}{\epsilon}} y\right]+\frac{1}{\epsilon} \int_{s}^{t} e^{(t-\sigma) \frac{B}{\epsilon}} G\left(u^{\epsilon}(\sigma), v^{\epsilon}(\sigma)\right) d \sigma \\
& +\frac{1}{\epsilon} \int_{0}^{s}\left[e^{(t-\sigma) \frac{B}{\epsilon}}-e^{(s-\sigma) \frac{B}{\epsilon}}\right] G\left(u^{\epsilon}(\sigma), v^{\epsilon}(\sigma)\right) d \sigma+\left[w^{\epsilon, B}(t)-w^{\epsilon, B}(s)\right]=: \sum_{k=1}^{4} I_{k}^{\epsilon}(t, s) .
\end{aligned}
$$

Proceeding as in the proof of Theorem 4.3, we have

$$
\left|I_{1}^{\epsilon}(t, s)\right|_{H} \leq c \epsilon^{-\frac{\alpha}{2}}(t-s)^{\frac{\alpha}{2}}|y|_{\alpha, 2} .
$$

Concerning $I_{2}^{\epsilon}(t, s)$, we have

$$
\left|I_{2}^{\epsilon}(t, s)\right|_{H} \leq \frac{c}{\epsilon} \int_{s}^{t} e^{-\lambda \frac{(t-\sigma)}{\epsilon}}\left(1+\left|u^{\epsilon}(\sigma)\right|_{H}+\left|v^{\epsilon}(\sigma)\right|_{H}\right) d \sigma
$$

and then, with a change of variables, according to (4.2) and (4.3) we get

$$
\mathbb{E}\left|I_{2}^{\epsilon}(t, s)\right|_{H} \leq c_{T} \int_{0}^{\frac{t-s}{\epsilon}} e^{-\lambda \sigma} d \sigma\left(1+|x|_{H}+|y|_{H}\right) \leq c_{T} \epsilon^{-\frac{\alpha}{2}}(t-s)^{\frac{\alpha}{2}}\left(1+|x|_{H}+|y|_{H}\right) .
$$

By proceeding with analogous arguments we prove that

$$
\mathbb{E}\left|I_{3}^{\epsilon}(t, s)\right|_{H} \leq c_{T} \epsilon^{-\frac{\alpha}{2}}(t-s)^{\frac{\alpha}{2}}\left(1+|x|_{H}+|y|_{H}\right) .
$$


Therefore, it remains to estimate $\mathbb{E}\left|I_{4}^{\epsilon}(t, s)\right|_{H}$. By straightforward computations, we have

$$
\begin{aligned}
& \mathbb{E}\left|I_{4}^{\epsilon}(t, s)\right|_{H}^{2}=\mathbb{E}\left|w^{\epsilon, B}(t)-w^{\epsilon, B}(s)\right|_{H}^{2} \\
& =\frac{1}{\epsilon} \int_{s}^{t}\left\|e^{(t-\sigma) B / \epsilon}\right\|_{2}^{2} d \sigma+\frac{1}{\epsilon} \int_{0}^{s}\left\|e^{(t-\sigma) B / \epsilon}-e^{(s-\sigma) B / \epsilon}\right\|_{2}^{2} d \sigma=: J_{1}^{\epsilon}+J_{2}^{\epsilon} .
\end{aligned}
$$

According to (2.6), with a change of variables we have

$$
J_{1}^{\epsilon} \leq c \int_{0}^{\frac{t-s}{\epsilon}} e^{-2 \lambda \sigma}(\sigma \wedge 1)^{-\gamma} d \sigma \leq c \epsilon^{-(1-\gamma)}(t-s)^{1-\gamma} .
$$

Concerning $J_{2}^{\epsilon}$, due to (2.2) and (4.8) for any $\eta \in[0,1 / 2]$ and $s, t>0$

$$
\left\|\left(e^{t B}-I\right) e^{s B}\right\|_{\mathcal{L}(H)} \leq c_{\eta}(t \wedge 1)^{\eta}(s \wedge 1)^{-\eta}
$$

Hence, thanks to (2.6), if $0<\eta<1-\gamma$, by proceeding with the same change of variables

$$
\begin{aligned}
& J_{2}^{\epsilon}=\frac{1}{\epsilon} \int_{0}^{s}\left\|\left[e^{(t-s) B / \epsilon}-I\right] e^{(s-\sigma) B / 2 \epsilon} e^{(s-\sigma) B / 2 \epsilon}\right\|_{2}^{2} d \sigma \\
& \leq \frac{c}{\epsilon}\left(\frac{t-s}{\epsilon} \wedge 1\right)^{\eta} \int_{0}^{s}\left(\frac{s-\sigma}{2 \epsilon} \wedge 1\right)^{-(\eta+\gamma)} e^{-\lambda \frac{s-\sigma}{2 \epsilon}} d \sigma \leq c \epsilon^{-\eta}(t-s)^{\eta},
\end{aligned}
$$

so that

$$
\mathbb{E}\left|I_{4}^{\epsilon}(t, s)\right|_{H} \leq\left(\mathbb{E}\left|I_{4}^{\epsilon}(t, s)\right|_{H}^{2}\right)^{\frac{1}{2}} \leq c \epsilon^{-(1-\gamma)}\left[(t-s)^{1-\gamma}+(t-s)^{\eta}\right] .
$$

Collecting together (4.16), (4.17), (4.18) and (4.19), we obtain

$$
\mathbb{E}\left|v^{\epsilon}(t)-v^{\epsilon}(s)\right|_{H} \leq c_{T} \epsilon^{-\frac{\alpha \vee(1-\gamma)}{2}}\left(1+|x|_{H}+|y|_{\alpha, 2}\right)\left[(t-s)^{\frac{\alpha}{2}}+(t-s)^{\eta}+(t-s)^{1-\gamma}\right],
$$

so that, from (4.15),

$$
\mathbb{E}\left|A u_{2}^{\epsilon}(t)\right|_{H} \leq c_{T}\left(1+|x|_{\alpha, 2}+|y|_{\alpha, 2}\right)\left(1+\epsilon^{-\frac{\alpha \vee(1-\gamma)}{2}}\right) .
$$

Together with (4.14), this yields (4.13).

\section{The averaging result}

Our aim here is proving the main result of the present paper. Namely, we are going to prove that for any fixed $T>0$ the sequence $\left\{u^{\epsilon}\right\}_{\epsilon>0} \subset C((0, T] ; H) \cap L^{\infty}(0, T ; H)$ converges in probability to the solution $\bar{u}$ of the averaged equation

$$
d u(t)=A u(t)+\bar{F}(u(t)), \quad u(0)=x .
$$

The non-linear coefficient $\bar{F}$ in the equation above is obtained by averaging the reaction coefficient $F$ appearing in the slow motion equation, with respect to the unique invariant measure $\mu^{x}$ of the fast motion equation (3.2), with frozen slow component $x$. More precisely,

$$
\bar{F}(x):=\int_{H} F(x, y) \mu^{x}(d y), \quad x \in H .
$$


Notice that, as the mapping $y \in H \mapsto F(x, y) \in H$ is Lipschitz-continuous, due to (3.19) the integral above is well defined. Moreover, as $\mu^{x}$ is ergodic, for any $h \in H$ we have

$$
\langle\bar{F}(x), h\rangle_{H}=\lim _{t \rightarrow \infty} \frac{1}{t} \int_{0}^{t}\left\langle F\left(x, v^{x, y}(s)\right), h\right\rangle_{H} d s, \quad \mathbb{P}-\text { a.s. }
$$

This implies that $\bar{F}$ is Lipschitz-continuous. Actually, as $F: H \times H \rightarrow H$ is Lipschitzcontinuous (with Lipschitz-constant $L_{f}$ ) and $v^{x, y}(t)$ is differentiable with respect to $x \in H$, with its derivative fulfilling (3.9), for any $x_{1}, x_{2} \in H$ and $t>0$ we have

$$
\begin{aligned}
& \frac{1}{t}\left|\int_{0}^{t}\left\langle F\left(x_{1}, v^{x_{1}, y}(s)\right)-F\left(x_{2}, v^{x_{2}, y}(s)\right), h\right\rangle_{H} d s\right| \\
& \leq \frac{L_{f}}{t} \int_{0}^{t}\left(\left|x_{1}-x_{2}\right|_{H}+\left|v^{x_{1}, y}(s)-v^{x_{2}, y}(s)\right|_{H}\right) d s|h|_{H} \\
& \leq L_{f}|h|_{H}\left(\left|x_{1}-x_{2}\right|_{H}+\sup _{\substack{x, y \in H \\
t \geq 0}}\left|D_{x} v^{x, y}(t)\right|_{\mathcal{L}(H)}\left|x_{1}-x_{2}\right|_{H}\right) \leq c\left(L_{f}+1\right)|h|_{H}\left|x_{1}-x_{2}\right|_{H} .
\end{aligned}
$$

Therefore, as (5.3) holds, we can conclude that $\bar{F}$ is Lipschitz-continuous, with

$$
[\bar{F}]_{\text {Lip }} \leq c\left(L_{f}+1\right)
$$

In particular, we have the following existence and uniqueness result for the averaged equation.

Proposition 5.1. Under Hypotheses 1, 20 and [3, equation (5.1) admits a unique mild solution $\bar{u} \in C((0, T] ; H) \cap L^{\infty}(0, T ; H)$, for any $T>0$ and $p \geq 1$ and for any initial datum $x \in H$.

As far as the differentiability of $\bar{F}$ is concerned, we have the following result.

Lemma 5.2. For any $h \in L^{\infty}(0, L)$, the mapping $\langle\bar{F}(\cdot), h\rangle_{H}: H \rightarrow \mathbb{R}$ is Fréchet differentiable and for any $k \in H$

$$
\begin{aligned}
& \langle D \bar{F}(x) k, h\rangle_{H}=\int_{H}\left\langle D_{x} F(x, y) k, h\right\rangle_{H} \mu^{x}(d y)+2 \int_{H}\left\langle U_{x}(x, y), k\right\rangle_{H}\langle F(x, y), h\rangle_{H} \mu^{x}(d y) \\
& -2 \int_{H}\left\langle U_{x}(x, y), k\right\rangle_{H} \mu^{x}(d y) \int_{H}\langle F(x, y), h\rangle_{H} \mu^{x}(d y),
\end{aligned}
$$

where $U_{x}(x, y)$ is the Fréchet derivative of the mapping $U(\cdot, y): H \rightarrow \mathbb{R}$ introduced in Subsection 3.3, for $y \in L^{\infty}(0, L)$ fixed.

Proof. It is immediate to check that for any $y \in L^{\infty}(0, L)$ the mapping

$$
x \in H \mapsto U(x, y)=\int_{0}^{1}\langle G(x, \theta y), y\rangle_{H} d \theta \in \mathbb{R},
$$


is Fréchet differentiable and for any $k \in H$

$$
\left\langle U_{x}(x, y), k\right\rangle_{H}=\int_{0}^{1}\left\langle G_{x}(x, \theta y) k, y\right\rangle_{H} d \theta,
$$

where $G_{x}(x, y)$ is the Gâteaux derivative of $G(\cdot, y)$ introduced in Section 2 ,

Then, if we define

$$
V(x, y):=\frac{1}{Z(x)} \exp 2 U(x, y), \quad x, y \in H
$$

by straightforward computations, for any $y \in L^{\infty}(0, L)$ the mapping $V(\cdot, y): H \rightarrow H$ is differentiable and we have

$$
D_{x} V(x, y)=2 V(x, y)\left[U_{x}(x, y)-\int_{H} U_{x}(x, z) \mu^{x}(d z)\right]=: 2 V(x, y) H(x, y) .
$$

Notice that, as we are assuming $\partial g / \partial \sigma_{1}(\xi, \sigma)$ to be uniformly bounded, we have

$$
\left|U_{x}(x, y)\right|_{H} \leq c|y|_{H}, \quad x, y \in H,
$$

so that thanks to (3.20) $D_{x} V(x, y)$ is well defined.

Now, according to (3.3), the measure $\mu^{x}$ is supported on $C([0, L])$, so that

$$
\langle\bar{F}(x), h\rangle_{H}=\int_{C([0, L])}\langle F(x, y), h\rangle_{H} \mu^{x}(d y) .
$$

Hence, if we set $\mu:=\mathcal{N}\left(0,(-B)^{-1} / 2\right)$, by differentiating under the sign of integral from (5.5) we have

$$
\begin{aligned}
& \langle D \bar{F}(x) k, h\rangle_{H}=\left\langle D \int_{C([0, L])}\langle F(x, y), h\rangle_{H} V(x, y) \mu(d y), k\right\rangle_{H} \\
& =\int_{C([0, L])}\left\langle D_{x} F(x, y) k, h\right\rangle_{H} \mu^{x}(d y)+2 \int_{C([0, L])}\langle F(x, y), h\rangle_{H} H(x, y) \mu^{x}(d y) \\
& =\int_{H}\left\langle D_{x} F(x, y) k, h\right\rangle_{H} \mu^{x}(d y)+2 \int_{H}\langle F(x, y), h\rangle_{H} H(x, y) \mu^{x}(d y),
\end{aligned}
$$

and recalling how $H(x, y)$ is defined, we can conclude the proof of the lemma.

Now, as $u^{\epsilon}$ is a mild solution of the slow motion equation (in fact it is a classical solution, as $u^{\epsilon}(t) \in D(A)$ for any $t>0$, and estimate (4.13) holds), for any $h \in D\left(A^{\star}\right)$

$$
\left\langle u^{\epsilon}(t), h\right\rangle_{H}=\langle x, h\rangle_{H}+\int_{0}^{t}\left\langle u^{\epsilon}(s), A^{\star} h\right\rangle_{H} d s+\int_{0}^{t}\left\langle F\left(u^{\epsilon}(s), v^{\epsilon}(s)\right), h\right\rangle_{H} d s, \quad t \geq 0 .
$$

Hence, we have

$$
\left\langle u^{\epsilon}(t), h\right\rangle_{H}=\langle x, h\rangle_{H}+\int_{0}^{t}\left\langle u^{\epsilon}(s), A^{\star} h\right\rangle_{H} d s+\int_{0}^{t}\left\langle\bar{F}\left(u^{\epsilon}(s)\right), h\right\rangle_{H} d s+R_{h}^{\epsilon}(t), \quad t \geq 0
$$


where the remainder $R_{h}^{\epsilon}(t)$ is clearly given by

$$
R_{h}^{\epsilon}(t):=\int_{0}^{t}\left\langle F\left(u^{\epsilon}(s), v^{\epsilon}(s)\right)-\bar{F}\left(u^{\epsilon}(s)\right), h\right\rangle_{H} d s, \quad t \geq 0 .
$$

Our purpose is proving that the remainder $R_{h}^{\epsilon}(t)$ converges to zero, as $\epsilon$ goes to zero. We will see that, thanks to Theorem 4.3, this will imply the averaging result.

Lemma 5.3. Assume Hypotheses 1, 2 and 3 and fix any $\alpha>0$. Then, for any $T>0$, $x, y \in W^{\alpha, 2}(0, L)$ and any $h \in H$

$$
\lim _{\epsilon \rightarrow 0} \sup _{t \in[0, T]} \mathbb{E}\left|R_{h}^{\epsilon}(t)\right|=0
$$

Proof. Fix $h \in L^{\infty}(0, L)$. For any $x, y \in H$ and $\epsilon>0$ we define

$$
\Phi_{h}^{\epsilon}(x, y):=\int_{0}^{\infty} e^{-c(\epsilon) t} P_{t}^{x}\left[\langle F(x, \cdot), h\rangle_{H}-\langle\bar{F}(x), h\rangle_{H}\right](y) d t
$$

where $c(\epsilon)$ is some positive constant, depending on $\epsilon>0$, to be chosen later on. As for any $y, z \in H$

$$
\left|\langle F(x, y), h\rangle_{H}-\langle F(x, z), h\rangle_{H}\right| \leq c|y-z|_{H}|h|_{H},
$$

for some constant $c$ independent of $x \in H$, we have that the mapping

$$
y \in H \rightarrow\langle F(x, y), h\rangle_{H}-\langle\bar{F}(x), h\rangle_{H} \in \mathbb{R}
$$

is Lipschitz-continuous and

$$
\left[\langle F(x, \cdot), h\rangle_{H}-\langle\bar{F}(x), h\rangle_{H}\right]_{\operatorname{Lip}} \leq c|h|_{H} .
$$

According to Theorem 3.8, this means that the function $\Phi_{h}^{\epsilon}(x, \cdot)$ is a strict solution of the problem

$$
c(\epsilon) \Phi_{h}^{\epsilon}(x, y)-\mathcal{L}^{x} \Phi_{h}^{\epsilon}(x, y)=\langle F(x, y), h\rangle_{H}-\langle\bar{F}(x), h\rangle_{H}, \quad y \in D(B) .
$$

Now, we prove uniform bounds in $\epsilon>0$ for $\Phi_{h}^{\epsilon}(x, y)$, for its first derivatives with respect to $y$ and $x$ and for $\operatorname{Tr}\left[D_{y}^{2} \Phi_{h}^{\epsilon}(x, y)\right]$. Due to (3.23) and (5.10), we have

$$
\left|P_{t}^{x}\langle F(x, \cdot), h\rangle_{H}(y)-\langle\bar{F}(x), h\rangle_{H}\right| \leq c\left(1+|x|_{H}+|y|_{H}\right) e^{-\delta t}|h|_{H},
$$

and then

$$
\left|\Phi_{h}^{\epsilon}(x, y)\right| \leq c \int_{0}^{\infty} e^{-c(\epsilon) t} e^{-\delta t} d t\left(1+|x|_{H}+|y|_{H}\right)|h|_{H} \leq \frac{c}{\delta}\left(1+|x|_{H}+|y|_{H}\right)|h|_{H},
$$

for some constant $c$ independent of $\epsilon>0$.

For the first derivative with respect to $y$, from (3.13) and (5.10) we get

$$
\left[P_{t}^{x}\langle F(x, \cdot), h\rangle_{H}-\langle\bar{F}(x), h\rangle_{H}\right]_{1} \leq c e^{-\delta t}|h|_{H},
$$


and then

$$
\begin{aligned}
& \left|D_{y} \Phi_{h}^{\epsilon}(x, y)\right|_{H}=\left|\int_{0}^{\infty} e^{-c(\epsilon) t} D_{y}\left[P_{t}^{x}\langle F(x, \cdot), h\rangle_{H}(y)-\langle\bar{F}(x), h\rangle_{H}\right] d t\right|_{H} \\
& \leq c \int_{0}^{\infty} e^{-c(\epsilon) t} e^{-\delta t} d t|h|_{H} \leq \frac{c}{\delta}|h|_{H}
\end{aligned}
$$

for a constant $c$ independent of $\epsilon>0$.

For the trace of $D_{y}^{2} \Phi_{h}^{\epsilon}(x, y)$, according to (3.15) we have

$$
\left|\operatorname{Tr}\left[D_{y}^{2} P_{t}^{x}\langle F(x, \cdot), h\rangle_{H}(y)\right]\right| \leq c(t \wedge 1)^{-\rho}\left(1+|x|_{H}+|y|_{H}\right)|h|_{H},
$$

for some $\rho<1$, and hence if $c(\epsilon) \leq 1$

$$
\begin{aligned}
& \left|\operatorname{Tr}\left[D_{y}^{2} \Phi^{\epsilon}(x, y)\right]\right| \leq \int_{0}^{\infty} e^{-c(\epsilon) t}\left|\operatorname{Tr}\left[D_{y}^{2}\left(P_{t}^{x}\langle F(x, \cdot), h\rangle_{H}(y)-\langle\bar{F}(x), h\rangle_{H}\right)\right]\right| d t \\
& \leq \int_{0}^{\infty} e^{-c(\epsilon) t}(t \wedge 1)^{-\rho} d t\left(1+|x|_{H}+|y|_{H}\right)|h|_{H} \leq \frac{c}{c(\epsilon)}\left(1+|x|_{H}+|y|_{H}\right)|h|_{H} .
\end{aligned}
$$

Next, concerning the regularity of $\Phi_{h}^{\epsilon}$ with respect to $x \in H$, we first compute the derivative of the mapping

$$
x \in H \mapsto P_{t}^{x}\langle F(x, \cdot), h\rangle_{H}(y)=\mathbb{E}\left\langle F\left(x, v^{x, y}(t)\right), h\right\rangle_{H} \in \mathbb{R} .
$$

As we are assuming that $h \in L^{\infty}$, we have that the mappings $\langle F(x, \cdot), h\rangle_{H}$ and $\langle F(\cdot, y), h\rangle_{H}$ are both Fréchet differentiable (see Section 2). Beside, as shown at the end of subsection 3.1, the process $v^{x, y}$ is differentiable with respect to $x$. Then, by differentiating above under the sign of integral, for any $k \in H$ we obtain

$$
\begin{aligned}
& \left\langle D_{x}\left[P_{t}^{x}\langle F(x, \cdot), h\rangle_{H}(y)\right], k\right\rangle_{H} \\
& =\mathbb{E}\left\langle D_{x} F\left(x, v^{x, y}(t)\right) k, h\right\rangle_{H}+\mathbb{E}\left\langle D_{y} F\left(x, v^{x, y}(t)\right) D_{x} v^{x, y}(t) k, h\right\rangle_{H} \\
& =P_{t}^{x}\left\langle D_{x} F(x, \cdot) k, h\right\rangle_{H}(y)+\mathbb{E}\left\langle D_{y} F\left(x, v^{x, y}(t)\right) D_{x} v^{x, y}(t) k, h\right\rangle_{H},
\end{aligned}
$$

so that, thanks to (2.10) and (3.9)

$$
\left|D_{x}\left[P_{t}^{x}\langle F(x, \cdot), h\rangle_{H}(y)\right]\right|_{H} \leq c L_{f}|h|_{H}, \quad t \geq 0 .
$$

Moreover, as shown in Lemma [5.2, the mapping $x \in H \mapsto\langle\bar{F}(x), h\rangle_{H} \in \mathbb{R}$ is Fréchet differentiable and, due to estimate (5.4), we have

$$
\left[\langle\bar{F}(x), h\rangle_{H}\right]_{1}=\left[\langle\bar{F}(x), h\rangle_{H}\right]_{\mathrm{Lip}} \leq c\left(L_{f}+1\right)|h|_{H} .
$$

Therefore

$$
\begin{aligned}
& \left|D_{x} \Phi_{h}^{\epsilon}(x, y)\right|_{H}=\left|\int_{0}^{\infty} e^{-c(\epsilon) t} D_{x}\left[P_{t}^{x}\langle F(x, \cdot), h\rangle_{H}(y)-\langle\bar{F}(x), h\rangle_{H}\right] d t\right|_{H} \\
& \leq c \int_{0}^{\infty} e^{-c(\epsilon) t} d t\left(L_{f}+1\right)|h|_{H}=\frac{c\left(L_{f}+1\right)}{c(\epsilon)}|h|_{H} .
\end{aligned}
$$


Next, for any $n \in \mathbb{N}$, we define $v_{n}^{\epsilon}:=P_{n} v^{\epsilon}$, where $P_{n}$ the projection of $H$ onto $\left\langle e_{1}, \ldots, e_{n}\right\rangle$ and $\left\{e_{k}\right\}_{k \in \mathbb{N}}$ is the complete orthonormal system, introduced in Hypothesis 2, which diagonalizes $B$. It is immediate to check that $v_{n}^{\epsilon}$ is a strong solution of equation

$$
d v_{n}^{\epsilon}(t)=\frac{1}{\epsilon}\left[B v_{n}^{\epsilon}(t)+P_{n} G\left(u^{\epsilon}(t), v^{\epsilon}(t)\right)\right] d t+\frac{1}{\sqrt{\epsilon}} P_{n} d w(t), \quad v_{n}^{\epsilon}(0)=P_{n} y .
$$

Moreover, according to Lemma 4.4, $u^{\epsilon}$ is a strong solution of the slow motion equation.

Therefore, we can apply Itô's formula to $\Phi_{h}^{\epsilon}\left(u^{\epsilon}(t), v_{n}^{\epsilon}(t)\right)$ and we get

$$
\begin{aligned}
& \Phi_{h}^{\epsilon}\left(u^{\epsilon}(t), v_{n}^{\epsilon}(t)\right)=\Phi_{h}^{\epsilon}\left(x, P_{n} y\right)+\int_{0}^{t}\left\langle D_{x} \Phi_{h}^{\epsilon}\left(u^{\epsilon}(s), v_{n}^{\epsilon}(s)\right), A u^{\epsilon}(s)+F\left(u^{\epsilon}(s), v^{\epsilon}(s)\right)\right\rangle_{H} d s \\
& +\frac{1}{\epsilon} \int_{0}^{t}\left\langle D_{y} \Phi_{h}^{\epsilon}\left(u^{\epsilon}(s), v_{n}^{\epsilon}(s)\right), B v_{n}^{\epsilon}(s)+P_{n} G\left(u^{\epsilon}(s), v^{\epsilon}(s)\right)\right\rangle_{H} d s \\
& +\frac{1}{2 \epsilon} \int_{0}^{t} \operatorname{Tr}\left[P_{n} D_{y}^{2} \Phi_{h}^{\epsilon}\left(u^{\epsilon}(s), v_{n}^{\epsilon}(s)\right)\right] d s+\frac{1}{\sqrt{\epsilon}} \int_{0}^{t}\left\langle D_{y} \Phi_{h}^{\epsilon}\left(u^{\epsilon}(s), v_{n}^{\epsilon}(s)\right), P_{n} d w(s)\right\rangle_{H},
\end{aligned}
$$

and hence

$$
\begin{aligned}
& \Phi_{h}^{\epsilon}\left(u^{\epsilon}(t), v_{n}^{\epsilon}(t)\right)=\Phi_{h}^{\epsilon}\left(x, P_{n} y\right)+\int_{0}^{t}\left\langle D_{x} \Phi_{h}^{\epsilon}\left(u^{\epsilon}(s), v_{n}^{\epsilon}(s)\right), A u^{\epsilon}(s)+F\left(u^{\epsilon}(s), v^{\epsilon}(s)\right)\right\rangle_{H} d s \\
& +\frac{1}{\epsilon} \int_{0}^{t} \mathcal{L}^{u^{\epsilon}(s)} \Phi_{h}^{\epsilon}\left(u^{\epsilon}(s), v_{n}^{\epsilon}(s)\right) d s+\frac{1}{\sqrt{\epsilon}} \int_{0}^{t}\left\langle D_{y} \Phi_{h}^{\epsilon}\left(u^{\epsilon}(s), v_{n}^{\epsilon}(s)\right), P_{n} d w(s)\right\rangle_{H} \\
& +\frac{1}{\epsilon} \int_{0}^{t}\left\langle D_{y} \Phi_{h}^{\epsilon}\left(u^{\epsilon}(s), v_{n}^{\epsilon}(s)\right),\left[P_{n} G\left(u^{\epsilon}(s), v^{\epsilon}(s)\right)-G\left(u^{\epsilon}(s), v_{n}^{\epsilon}(s)\right)\right]\right\rangle_{H} d s \\
& +\frac{1}{2 \epsilon} \int_{0}^{t} \operatorname{Tr}\left[\left(P_{n}-I\right) D_{y}^{2} \Phi_{h}^{\epsilon}\left(u^{\epsilon}(s), v_{n}^{\epsilon}(s)\right)\right] d s .
\end{aligned}
$$

Recalling that $\Phi_{h}^{\epsilon}(x, \cdot)$ is a strict solution of the elliptic equation (5.11), for any $s \geq 0$ we have

$$
\mathcal{L}^{u^{\epsilon}(s)} \Phi_{h}^{\epsilon}\left(u^{\epsilon}(s), v_{n}^{\epsilon}(s)\right)=c(\epsilon) \Phi_{h}^{\epsilon}\left(u^{\epsilon}(s), v_{n}^{\epsilon}(s)\right)-\left(\left\langle F\left(u^{\epsilon}(s), v_{n}^{\epsilon}(s)\right), h\right\rangle_{H}-\left\langle\bar{F}\left(u^{\epsilon}(s)\right), h\right\rangle_{H}\right) .
$$

Then, multiplying both sides of (5.16) by $\epsilon$,

$$
\begin{aligned}
& R_{\epsilon}(t)=\int_{0}^{t}\left[\left\langle F\left(u^{\epsilon}(s), v^{\epsilon}(s)\right), h\right\rangle_{H}-\left\langle\bar{F}\left(u^{\epsilon}(s)\right), h\right\rangle_{H}\right] d s=c(\epsilon) \int_{0}^{t} \Phi_{h}^{\epsilon}\left(u^{\epsilon}(s), v_{n}^{\epsilon}(s)\right) d s \\
& +\sqrt{\epsilon} \int_{0}^{t}\left\langle D_{y} \Phi_{h}^{\epsilon}\left(u^{\epsilon}(s), v_{n}^{\epsilon}(s)\right), P_{n} d w(s)\right\rangle_{H}-\epsilon\left[\Phi_{h}^{\epsilon}\left(u^{\epsilon}(t), v_{n}^{\epsilon}(t)\right)-\Phi_{h}^{\epsilon}(x, y)\right] \\
& +\epsilon \int_{0}^{t}\left\langle D_{x} \Phi_{h}^{\epsilon}\left(u^{\epsilon}(s), v_{n}^{\epsilon}(s)\right), A u^{\epsilon}(s)+F\left(u^{\epsilon}(s), v^{\epsilon}(s)\right)\right\rangle_{H} d s+H^{n, \epsilon}(t),
\end{aligned}
$$


where

$$
\begin{aligned}
& H^{n, \epsilon}(t):=\int_{0}^{t}\left\langle D_{y} \Phi_{h}^{\epsilon}\left(u^{\epsilon}(s), v_{n}^{\epsilon}(s)\right),\left[P_{n} G\left(u^{\epsilon}(s), v^{\epsilon}(s)\right)-G\left(u^{\epsilon}(s), v_{n}^{\epsilon}(s)\right)\right]\right\rangle_{H} d s \\
& +\frac{1}{2} \int_{0}^{t} \operatorname{Tr}\left[\left(P_{n}-I\right) D_{y}^{2} \Phi_{h}^{\epsilon}\left(u^{\epsilon}(s), v_{n}^{\epsilon}(s)\right)\right] d s+\int_{0}^{t}\left\langle F\left(u^{\epsilon}(s), v^{\epsilon}(s)\right)-F\left(u^{\epsilon}(s), v_{n}^{\epsilon}(s)\right), h\right\rangle_{H} d s .
\end{aligned}
$$

Thanks to (5.12), (5.13) and (5.15), this yields

$$
\begin{aligned}
& \left|R_{h}^{\epsilon}(t)\right| \leq c\left(\frac{\epsilon}{c(\epsilon)}+c(\epsilon)\right) \int_{0}^{t}\left(1+\left|u^{\epsilon}(s)\right|_{H}+\left|v^{\epsilon}(s)\right|_{H}+\left|v_{n}^{\epsilon}(s)\right|_{H}\right) d s|h|_{H} \\
& +c \frac{\epsilon}{c(\epsilon)} \int_{0}^{t}\left|A u^{\epsilon}(s)\right|_{H} d s|h|_{H}+\sqrt{\epsilon}\left|\int_{0}^{t}\left\langle D_{y} \Phi_{h}^{\epsilon}\left(u^{\epsilon}(s), v_{n}^{\epsilon}(s)\right), P_{n} d w(s)\right\rangle_{H}\right| \\
& +\epsilon\left(1+\left|u^{\epsilon}(t)\right|_{H}+\left|v_{n}^{\epsilon}(t)\right|_{H}+|x|_{H}+|y|_{H}\right)|h|_{H}+\left|H^{n, \epsilon}\right|,
\end{aligned}
$$

and hence, by taking expectation, due to (4.2), (4.3), (4.13) and (5.13), for any $n \in \mathbb{N}$

$$
\begin{aligned}
& \mathbb{E}\left|R_{h}^{\epsilon}(t)\right| \leq c_{T}\left(\frac{\epsilon}{c(\epsilon)}+c(\epsilon)+\epsilon\right)\left(1+|x|_{H}+|y|_{H}\right)|h|_{H} \\
& +c_{T} \frac{\epsilon}{c(\epsilon)}\left(1+\epsilon^{-\frac{\alpha \vee(1-\gamma)}{2}}\right)\left(1+|x|_{\alpha, 2}+|y|_{\alpha, 2}\right)|h|_{H}+c_{T} \sqrt{\epsilon}|h|_{H}+\mathbb{E}\left|H^{n, \epsilon}\right| .
\end{aligned}
$$

Now, thanks to estimates (4.2), (4.3), (5.13) and (5.14), by using the dominated convergence theorem, for any $\epsilon>0$ we have

$$
\lim _{n \rightarrow \infty} \mathbb{E}\left|H^{n, \epsilon}\right|=0 .
$$

This means that if we take $c(\epsilon)=\epsilon^{\delta}$, with $0<\delta<1-[\alpha \vee(1-\gamma)] / 2$, and $n_{\epsilon} \in \mathbb{N}$ such that $\mathbb{E}\left|H^{n_{\epsilon}, \epsilon}\right| \leq \epsilon$, it follows

$$
\begin{aligned}
& \sup _{t \in[0, T]} \mathbb{E}\left|R_{h}^{\epsilon}(t)\right| \leq c_{T}\left(\frac{\epsilon}{c(\epsilon)}+c(\epsilon)+\epsilon\right)\left(1+|x|_{H}+|y|_{H}\right)|h|_{H} \\
& +c_{T} \frac{\epsilon}{c(\epsilon)}\left(1+\epsilon^{-\frac{\alpha \vee(1-\gamma)}{2}}\right)\left(1+|x|_{\alpha, 2}+|y|_{\alpha, 2}\right)|h|_{H}+c_{T} \sqrt{\epsilon}|h|_{H}+\mathbb{E}\left|H^{n_{\epsilon}, \epsilon}\right| \\
& \leq c_{T} e^{\rho}\left(1+|x|_{\alpha, 2}+|y|_{\alpha, 2}\right)|h|_{H}
\end{aligned}
$$

for some $\rho>0$. This immediately yields (5.8) for $h \in L^{\infty}(0, L)$.

Now, if $h \in H$ we fix a sequence $\left\{h_{n}\right\}_{n \in \mathbb{N}} \subset L^{\infty}(0, L)$ converging to $h$ in $H$ and such that $\left|h_{h}\right|_{H} \leq|h|_{H}$. As

$$
\sup _{t \in[0, T]} \mathbb{E}\left|R_{h_{n}}^{\epsilon}(t)\right| \leq c_{T} \epsilon^{\rho}\left(1+|x|_{\alpha, 2}+|y|_{\alpha, 2}\right)\left|h_{n}\right|_{H},
$$

we obtain (5.8) also in the general case. 
Once we have proved the key Lemma 5.3, we can prove the main result of the paper, the convergence of the solution of the slow motion equation to the solution of the averaged equation.

Theorem 5.4. Assume that $x, y \in W^{\alpha, 2}(0, L)$, for some $\alpha>0$. Then, under Hypotheses 1. 2 and [, for any $T>0$ and $\eta>0$ we have

$$
\lim _{\epsilon \rightarrow 0} \mathbb{P}\left(\sup _{t \in[0, T]}\left|u^{\epsilon}(t)-\bar{u}(t)\right|_{H}>\eta\right)=0,
$$

where $\bar{u}$ is the solution of the averaged equation (5.1).

Proof. Due to Theorem 4.3, the sequence $\left\{\mathcal{L}\left(u^{\epsilon}\right)\right\}_{\epsilon>0}$ is tight in $C((0, T] ; H) \cap L^{\infty}(0, T ; H)$, and then as a consequence of the Skorokhod theorem, for any two sequences $\left\{\epsilon_{n}\right\}_{n \in \mathbb{N}}$ and $\left\{\epsilon_{m}\right\}_{m \in \mathbb{N}}$ converging to zero, there exist subsequences $\left\{\epsilon_{n(k)}\right\}_{k \in \mathbb{N}}$ and $\left\{\epsilon_{m(k)}\right\}_{k \in \mathbb{N}}$ and a sequence of random elements

$$
\left\{\rho_{k}\right\}_{k \in \mathbb{N}}:=\left\{\left(u_{1}^{k}, u_{2}^{k}\right)\right\}_{k \in \mathbb{N}},
$$

in $\mathcal{C}:=\left[C((0, T] ; H) \cap L^{\infty}(0, T ; H)\right]^{2}$, defined on some probability space $(\hat{\Omega}, \hat{\mathcal{F}}, \hat{\mathbb{P}})$, such that the law of $\rho_{k}$ coincides with the law of $\left(u^{\epsilon_{n(k)}}, u^{\epsilon_{m(k)}}\right)$, for each $k \in \mathbb{N}$, and $\rho_{k}$ converges $\hat{\mathbb{P}}_{\text {-a.s. }}$ to some random element $\rho:=\left(u_{1}, u_{2}\right) \in \mathcal{C}$. By a well known argument due to Gyöngy and Krylov (see [10]), if we show that $u_{1}=u_{2}$, then we can conclude that there exists some $u \in C((0, T] ; H) \cap L^{\infty}(0, T ; H)$ such that the whole sequence $\left\{u^{\epsilon}\right\}_{\epsilon>0}$ converges to $u$ in probability.

For any $k \in \mathbb{N}$ and $i=1,2$ we define

$$
R_{i}^{k}(t):=\left\langle u_{i}^{k}(t), h\right\rangle_{H}-\langle x, h\rangle_{H}-\int_{0}^{t}\left\langle u_{1}^{k}(s), A^{\star} h\right\rangle_{H} d s-\int_{0}^{t}\left\langle\bar{F}\left(u_{i}^{k}(s)\right), h\right\rangle_{H} d s .
$$

As $\mathcal{L}\left(u_{1}^{k}\right)=\mathcal{L}\left(u^{\epsilon_{n(k)}}\right)$ and $\mathcal{L}\left(u_{1}^{k}\right)=\mathcal{L}\left(u^{\epsilon_{m(k)}}\right)$, according to (5.8) we have

$$
\lim _{k \rightarrow \infty} \sup _{t \in[0, T]} \hat{\mathbb{E}}\left|R_{i}^{k}(t)\right|=0,
$$

so that, as the sequences $\left\{u_{1}^{k}\right\}_{k \in \mathbb{N}}$ and $\left\{u_{2}^{k}\right\}_{k \in \mathbb{N}}$ converge $\hat{\mathbb{P}}$-a.s. in $C((0, T] ; H) \cap L^{\infty}(0, T ; H)$ respectively to $u_{1}$ and $u_{2}$, by taking the limit for some $\left\{k_{i}(n)\right\} \subseteq\{k\}$ going to infinity in (5.18), we have that both $u_{1}$ and $u_{2}$ fulfill the equation

$$
\langle u(t), h\rangle_{H}=\langle x, h\rangle_{H}+\int_{0}^{t}\left\langle u(s), A^{\star} h\right\rangle_{H} d s+\int_{0}^{t}\langle\bar{F}(u(s)), h\rangle_{H} d s,
$$

for any $h \in D\left(A^{\star}\right)$, and then they coincide with the unique solution of the averaged equation (5.1).

As we have recalled before, this implies that the sequence $\left\{u^{\epsilon}\right\}_{\epsilon>0}$ converges in probability to some $u \in C([0, L] ; H)$, and, by using again a uniqueness argument, such $u$ has to coincide with the solution $\bar{u}$ of equation (5.1). 


\section{Some remarks on the case of space dimension $d>1$}

In the case of space dimension $d=1$, the fast equation (3.2) with frozen slow component $x \in H$ is a gradient system and hence its unique invariant measure $\mu^{x}$ admits a density $V(x, y)$ with respect to the Gaussian measure $\mathcal{N}\left(0,(-B)^{-1} / 2\right)$. This allows to prove in Lemma 5.2 that for any $h \in L^{\infty}(0, L)$ the mapping

$$
x \in H \mapsto\langle\bar{F}(x), h\rangle_{H} \in \mathbb{R}
$$

is Fréchet differentiable and also allows to compute its derivative.

In space dimension $d>1$, in order to have function-valued solutions to system (1.7) we have to take a noise colored in space, and hence the fast equation is no more a gradient system. For this reason we cannot say anything about the differentiability of mapping (6.1) and hence we cannot say anything about the differentiability with respect to $x \in H$ of the mapping $\Phi_{h}^{\epsilon}(x, y)$ introduced in (5.9). Nevertheless, under suitable assumptions on the noise in the fast equation, it is possible to prove a result analogous to that proved in Lemma 5.3 and hence to get averaging.

Instead of working in the interval $(0, L)$, now we work in a bounded open set $D \subset \mathbb{R}^{d}$, with $d>1$, having a regular boundary. In the fast motion equation we take a noise of the following form

$$
w^{Q}(t, \xi)=\sum_{k=1}^{\infty} Q e_{k}(\xi) \beta_{k}(t), \quad t \geq 0, \quad \xi \in D,
$$

and we assume that the operators $B$ and $Q$ satisfy the following conditions.

Hypothesis 4. 1. There exists a complete orthonormal system $\left\{e_{k}\right\}_{k \in \mathbb{N}}$ in $H$ and two positive sequences $\left\{\alpha_{k}\right\}_{k \in \mathbb{N}}$ and $\left\{\lambda_{k}\right\}_{k \in \mathbb{N}}$ such that $B e_{k}=-\alpha_{k} e_{k}$ and $Q e_{k}=\lambda_{k} e_{k}$ and, for some $\gamma<1$,

$$
\sum_{k=1}^{\infty} \frac{\lambda_{k}^{2}}{\alpha_{k}^{1-\gamma}}<\infty
$$

2. There exists $\lambda>0$ such that $\alpha_{k} \geq \lambda$, for any $k \in \mathbb{N}$.

3. There exists $\eta<1 / 2$ such that

$$
\inf _{k \in \mathbb{N}} \lambda_{k} \alpha_{k}^{\eta}>0
$$

Notice that, as $\alpha_{k} \sim k^{2 / d}$, the conditions above imply that we have to work with $d \leq 3$. Under Hypothesis 4 and Hypotheses 2 and 3 (with obvious changes due to the passage from $d=1$ to $d \geq 1$ ) system (1.7) admits a unique mild solution $\left(u^{\epsilon}, v^{\epsilon}\right) \in \mathcal{C}_{T, p} \times \mathcal{C}_{T, p}$, for any $\epsilon>0, p \geq 1$ and $T>0$, and for any fixed slow component $x \in H$ the fast equation (3.2) admits a unique mild solution $v^{x, y} \in \mathcal{C}_{T, p}$, fulfilling (3.3) and (3.4). As in the one dimensional case, the process $v^{x, y}$ is three times differentiable with respect to $y \in H$ and once with respect to $x \in H$ and estimates analogous to (3.7), (3.8) and (3.9) hold (for all details see [4]).

The fast transition semigroup $P_{t}^{x}$ maps $C_{b}(H)$ into itself and $\operatorname{Lip}(H)$ into itself and (3.11) holds. Moreover, it has a smoothing effect and maps $B_{b}(H)$ into $C_{b}^{3}(H)$ and estimates (3.12), (3.13) and (3.14) are still true, with the singularity $(t \wedge 1)^{(j-i) / 2}$ replaced by $(t \wedge 1)^{(j-i)(\eta+1 / 2)}$. 
As far as the asymptotic behavior of the fast semigroup is concerned, it admits a unique invariant measure $\mu^{x}$ which is strongly mixing and fulfills (3.20), (3.21) (with the singularity $(t \wedge 1)^{1 / 2}$ replaced by the singularity $\left.(t \wedge 1)^{-(\eta+1 / 2)}\right)$ and (3.23). But, as we have said before, as equation (3.2) is not of gradient type, we do not have any explicit expression for the measure $\mu^{x}$.

All uniform bounds for $u^{\epsilon}$ and $v^{\epsilon}$ proved in Section 4 are still valid, so that the family of probability measures $\left\{\mathcal{L}\left(u^{\epsilon}\right)\right\}_{\epsilon \in(0,1)}$ is tight in $C((0, T] ; H) \cap L^{\infty}(0, T ; H)$. This means that in order to have averaging in this multidimensional case it suffices to prove Lemma 5.3 . The proof in this case follows the same lines as in the one dimensional case, but it requires some extra approximation arguments. Actually, one has to introduce the approximating problems

$$
d v_{n}^{\epsilon}(t)=\frac{1}{\epsilon}\left[B_{n} v_{n}^{\epsilon}+G_{n}\left(u^{\epsilon}(t), v_{n}^{\epsilon}(t)\right)\right] d t+\frac{1}{\sqrt{\epsilon}} Q_{n} d w(t), \quad v_{n}^{\epsilon}(0)=P_{n} y
$$

and

$$
d v_{n}^{x, y}(t)=\left[B_{n} v_{n}^{x, y}+G_{n}\left(x, v_{n}^{x, y}(t)\right)\right] d t+Q_{n} d w(t), \quad v_{n}^{x, y}(0)=P_{n} y,
$$

where $B_{n} x:=B P_{n} x, Q_{n} x:=Q P_{n} x$ and $G_{n}(x, y):=P_{n} G\left(x, P_{n} x\right)$, for any $n \in \mathbb{N}$ and $x, y \in H$. As the operators $B_{n}$ and $Q_{n}$ fulfill Hypothesis 4 and $G_{n}$ has the same regularity properties of $G$, all properties satisfied by $v^{\epsilon}, v^{x, y}$ and $P_{t}^{x}$ are still valid for $v_{n}^{\epsilon}, v_{n}^{x, y}$ and for the transition semigroup $P_{t}^{n, x}$ associated with (6.3). Moreover, all estimates for $v_{n}^{x, y}$ and $P_{t}^{n, x}$ are uniform with respect to $n \in \mathbb{N}$, and for each fixed $\epsilon>0$ and $x, y \in H$

$$
\lim _{n \rightarrow \infty} \mathbb{E} \sup _{t \geq 0}\left|v_{n}^{\epsilon}(t)-v^{\epsilon}(t)\right|_{H}^{2}=0
$$

and

$$
\lim _{n \rightarrow \infty} \mathbb{E} \sup _{t \geq 0}\left|v_{n}^{x, y}(t)-v^{x, y}(t)\right|_{H}^{2}=0 .
$$

Clearly, equation (6.3) shows the same long-time behavior as equation (3.2). Then for any $n \in \mathbb{N}$ there exists a unique invariant measure $\mu^{n, x}$ for the semigroup $P_{t}^{n, x}$, which fulfills all properties described for $\mu^{x}$, with all estimates uniform with respect to $n \in \mathbb{N}$.

Next, we define

$$
\bar{F}_{n}(x):=\int_{H} F(x, y) \mu^{n, x}(d y), \quad x \in H .
$$

As for $\bar{F}$, we obtain that all $\bar{F}_{n}: H \rightarrow H$ are Lipschitz-continuous and

$$
\sup _{n \in \mathbb{N}}\left[\bar{F}_{n}\right]_{\text {Lip }} \leq c L_{f}
$$

Moreover, for any $x \in H$

$$
\lim _{n \rightarrow \infty}\left|\bar{F}_{n}(x)-\bar{F}(x)\right|_{H}=0
$$

For any $n \in \mathbb{N}$ we define

$$
H_{n}(x):=\int_{\mathbb{R}^{n}} \bar{F}_{n}\left(P_{n} x-\sum_{k=1}^{n} \xi_{k} e_{k}\right) \rho_{n}(\xi) d \xi, \quad x \in H,
$$


where $\rho_{n}: \mathbb{R}^{n} \rightarrow \mathbb{R}$ is a $C^{1}$ mapping having support in $B_{\mathbb{R}^{n}}(0,1 / n)$ and having total mass equal 1. All mappings $H_{n}$ are in $C^{1}(H ; H)$ and

$$
\lim _{n \rightarrow \infty}\left|\bar{F}_{n}(x)-H_{n}(x)\right|_{H}=0, \quad x \in H
$$

Moreover, due to (6.6), we have

$$
\left|\bar{F}_{n}(x)-H_{n}(x)\right|_{H} \leq c\left(1+|x|_{H}\right), \quad \sup _{n \in \mathbb{N}}\left[H_{n}\right]_{\operatorname{Lip}(H)}<\infty .
$$

Then, in the proof of Lemma 5.3 we introduce the following correction function

$$
\Phi_{n}^{\epsilon}(x, y):=\int_{0}^{\infty} e^{-c(\epsilon) t} P_{t}^{n, x}\left[\langle F(x, \cdot), h\rangle_{H}-\left\langle H_{n}(x), h\right\rangle_{H}\right](y) d t
$$

As in the one dimensional case, we have that the function $\Phi_{n}^{\epsilon}(x, \cdot)$ is a strict solution of the problem

$$
c(\epsilon) \Phi_{n}^{\epsilon}(x, y)-\mathcal{L}^{n, x} \Phi_{n}^{\epsilon}(x, y)=\langle F(x, y), h\rangle_{H}-\left\langle H_{n}(x), h\right\rangle_{H}, \quad y \in H
$$

where $\mathcal{L}^{n, x}$ is the Kolmogorov operator associated with the approximating fast equation (6.3).

Concerning the regularity of $\Phi_{n}^{\epsilon}$ with respect to $y$, we proceed as in the proof of Lemma 5.3 and all estimates are uniform with respect to $n \in \mathbb{N}$. As far as regularity in $x$ is concerned, we also proceed as in the one dimensional case, by noticing that the mapping $x \in H \mapsto\left\langle H_{n}(x), h\right\rangle_{H} \in \mathbb{R}$ is Fréchet differentiable and, due to estimate (6.8), the $C^{1}$-norm is uniformly bounded in $n \in \mathbb{N}$, that is

$$
\sup _{n \in \mathbb{N}}\left[\left\langle H_{n}(\cdot), h\right\rangle_{H}\right]_{1}=\sup _{n \in \mathbb{N}}\left[\left\langle H_{n}(\cdot), h\right\rangle_{H}\right]_{\text {Lip }}=c|h|_{H}<\infty .
$$

This implies an estimate for $D_{x} \Phi_{n}^{\epsilon}$, which is uniform with respect to $n \in \mathbb{N}$.

Next, as in the proof of Lemma 5.3 we apply Itô's formula to $\Phi_{n}^{\epsilon}\left(u^{\epsilon}(t), v_{n}^{\epsilon}(t)\right)$ and, by some estimates not different from those already used, by taking $c(\epsilon)=\epsilon^{\delta}$, for some $\delta>0$ we obtain

$$
\begin{aligned}
& \mathbb{E}\left|\int_{0}^{t}\left[\left\langle F\left(u^{\epsilon}(s), v_{n}^{\epsilon}(s)\right), h\right\rangle_{H}-\left\langle H_{n}\left(u^{\epsilon}(s)\right), h\right\rangle_{H}\right] d s\right| \\
& \leq c_{T} \epsilon^{\delta^{\prime}}\left(1+|x|_{\alpha, 2}+|y|_{\alpha, 2}\right)|h|_{H}+\int_{0}^{T} \mathbb{E}\left|\bar{F}_{n}\left(u^{\epsilon}(s)\right)-H_{n}\left(u^{\epsilon}(s)\right)\right|_{H} d s|h|_{H},
\end{aligned}
$$

for some $\delta^{\prime}>0$. Due to (6.7) and (6.8), this allow to conclude that (5.8) holds.

\section{References}

[1] V. I. Arnold, V. V. Kozlov, A. I. Neishtadt, Mathematical aspects of Classical and Celestial mechanics. [Dynamical systems. III], third edition, Encyclopaedia of Mathematical Sciences, Springer-Verlag, Berlin, 2006. 
[2] N. N. Bogoliubov, Y. A. Mitropolsky, Asymptotic methods in the theory of Non-Linear oscillations, Gordon and Breach Science Publishers, New York 1961.

[3] M. Brin, M. I. Freidlin, On stochastic behavior of perturbed Hamiltonian systems, Ergodic Theory and Dynamical Systems 20 (2000), pp. 55-76.

[4] S. Cerrai, Second order PDE's in finite and infinite dimension. A probaBILISTIC APPROACH, $\mathrm{x}+330 \mathrm{pp}$. Lecture Notes in Mathematics 1762, Springer Verlag (2001).

[5] G. Da Prato, J. Zabczyk, Stochastic Equations in Infinite Dimensions, Cambridge University Press, Cambridge (1992).

[6] G. Da Prato, J. Zabczyk, Ergodicity for Infinite Dimensional Systems, London Mathematical Society, Lecture Notes Series 229, Cambridge University Press, Cambridge (1996).

[7] M. I. Freidlin, On stable oscillations and equilibrium induced by small noise, Journal of Statistical Physics 103 (2001), pp. 283-300.

[8] M. I. Freidlin, A. D. Wentzell, Random Perturbations of Dynamical Systems, Second Edition, Springer Verlag (1998).

[9] M. I. Freidlin, A. D. Wentzell, Long-time behavior of weakly coupled oscillators, Journal of Statistical Physics 123 (2006), pp. 1311-1337.

[10] I. Gyöngy, N.V. Krylov, Existence of strong solutions for Itô's stochastic equations via approximations, Probability Theory and Related Fields 103 (1996), pp. 143-158.

[11] R. Z. Khasminskii, On the principle of averaging the Itô's stochastic differential equations (Russian), Kibernetika 4 (1968), pp. 260-279.

[12] Y. Kifer, Some recent advances in averaging, Modern dynamical systems and applications, pp. 385-403, Cambridge Univ. Press, Cambridge, 2004.

[13] Y. Kifer, Diffusion approximation for slow motion in fully coupled averaging, Probability Theory and Related Fields 129 (2004), pp. 157-181.

[14] Y. Kifer, Averaging and climate models, Stochastic climate models (Chorin, 1999), pp. 171-188, Progress in Probability 49, Birkhuser, Basel, 2001.

[15] Y. Kifer, Stochastic versions of Anosov's and Neistadt's theorems on averaging, Stochastics and Dynamics 1 (2001), pp. 1-21.

[16] S. B. Kuksin, A. L. Piatnitski, Khasminski-Whitman averaging for randonly perturbed KdV equations, Preprint (2006)

[17] B. Maslowski, J. Seidler, I. Vrkoč, An averaging principle for stochastic evolution equations. II, Mathematica Bohemica 116 (1991), pp. 191-224. 
[18] A. Neishtadt, Averaging in multyfrequency systems, Soviet Physics Doktagy 21 (1976), pp. $80-82$.

[19] G. C. Papanicolaou, D. Stroock, S. R. S. Varadhan, Martingale approach to some limit theorems, in Papers from the Duke Turbolence Conference (Duke Univ. Duhram, N.C. 1976), Paper 6, ii+120 pp. Duke Univ. Math. Ser. Vol. III, Duke Univ. Duhram, N.C. (1977).

[20] J. Seidler, I. Vrkoč, An averaging principle for stochastic evolution equations, Časopis Pěst. Mat. 115 (1990), pp. 240-263.

[21] A. Y. Veretennikov, On the averaging principle for systems of stochastic differential equation, Mathematics of the USSR-Sbornik 69 (1991), pp. 271-284.

[22] V. M. Volosov, Averaging in systems of ordinary differential equations, Russian Matehmatical Surveys 17 (1962), pp. 1-126. 